\title{
ON A QUADRATIC-TRIGONOMETRIC FUNCTIONAL EQUATION AND SOME APPLICATIONS
}

\author{
J. K. CHUNG, B. R. EBANKS, C. T. NG AND P. K. SAHOO
}

\begin{abstract}
Our main goal is to determine the general solution of the functional equation

$$
\begin{gathered}
f_{1}(x y)+f_{2}\left(x y^{-1}\right)=f_{3}(x)+f_{4}(y)+f_{5}(x) f_{6}(y), \\
f_{i}(t x y)=f_{i}(t y x) \quad(i=1,2)
\end{gathered}
$$

where $f_{i}$ are complex-valued functions defined on a group. This equation contains, among others, an equation of $\mathbf{H}$. Swiatak whose general solution was not known until now and an equation studied by K.S. Lau in connection with a characterization of Rao's quadratic entropies. Special cases of this equation also include the Pexider, quadratic, d'Alembert and Wilson equations.
\end{abstract}

\section{INTRODUCTION}

In connection with the characterization of quadratic entropies of C.R. Rao, Lau [9] obtained the solution of the functional equation

$$
f\left(\frac{x+y}{2}\right)+f\left(\frac{x-y}{2}\right)=2 f\left(\frac{x}{2}\right)+2 f\left(\frac{y}{2}\right)+\lambda f(x) f(y)
$$

assuming $f$ to be an even, continuous, nonnegative function defined on the closed interval $[-1,1]$, with $f(0)=0$ and $f(1)=1$ and infinitely differentiable on the open interval ] $-1,1[$. The constant $\lambda$ in (1.1) is an arbitrary a priori chosen nonnegative real number. Lau showed that under the above assumptions the solution of $(1.1)$ is

$$
f(x)=x^{2}, \quad x \in[-1,1] .
$$

We have shown elsewhere [3] that to obtain the above solution the assumptions such as evenness, nonnegativity and the infinite differentiability are redundant. In the present paper, we solve the generalization

$$
f(x+y)+f(x-y)=2 f(x)+2 f(y)+\lambda f(2 x) f(2 y)
$$

of (1.1) on groups, without any regularity assumption about $f$. This equation is a special case of the functional equation

$$
f(x+y)+f(x-y)=2 f(x)+2 f(y)+g(x) g(y),
$$

Received by the editors March 23, 1992.

1991 Mathematics Subject Classification. Primary 39B52, 39B32.

Key words and phrases. Pexider equation, d'Alembert equation, convolution type functional equations, additive map, exponential map, quadratic entropy. 
which was introduced by Swiatak [12] as a generalization of the parallelogram law (for which $g=0$ ). Swiatak found the general solution of (SE) for $x, y \in$ $\mathbf{G}$, an abelian group, where $f, g: \mathbf{G} \rightarrow \mathbf{K}$, a commutative ring without zero divisors, under the additional hypothesis that $g(e) \neq 0$ (where $e$ is the identity element of $\mathbf{G}$ ). We remove this additional hypothesis on $g$ to find the general solution of (SE) on groups, while requiring $\mathbf{K}$ to be a field.

The solution of (SE) is found in turn by specializing the general solution of the more general equation

$$
f_{1}(x+y)+f_{2}(x-y)=f_{3}(x)+f_{4}(y)+f_{5}(x) f_{6}(y)
$$

for all $x, y \in \mathbf{G}$ and $f_{i}: \mathbf{G} \rightarrow \mathbf{K}(i=1,2,3,4,5,6)$, where $\mathbf{G}$ is a group and $\mathbf{K}$ is a quadratically closed commutative field with characteristic different from 2 and 3. Equation (1.2) contains many classical functional equations and readers should refer to $[1,2,4,5,7,8,10,11$ and 14]. We shall not assume $\mathbf{G}$ to be abelian, so we write (1.2) as

$$
f_{1}(x y)+f_{2}\left(x y^{-1}\right)=f_{3}(x)+f_{4}(y)+f_{5}(x) f_{6}(y) \quad(x, y \in \mathbf{G})
$$

but we will suppose

$$
f_{i}(t x y)=f_{i}(t y x), \quad i=1,2 \quad(t, x, y \in \mathbf{G}) .
$$

The condition (FC) was first considered by Kannappan in [7] while studying the cosine functional equation on groups. It is the condition that a function can be factored through the abelianization of $\mathbf{G}$.

Interchanging $y$ with $y^{-1}$ in (FE) we obtain

$$
f_{1}\left(x y^{-1}\right)+f_{2}(x y)=f_{3}(x)+f_{4}\left(y^{-1}\right)+f_{5}(x) f_{6}\left(y^{-1}\right) .
$$

Adding and subtracting it to and from (FE), we obtain respectively

$$
f(x y)+f\left(x y^{-1}\right)=p(x)+q(y)+g(x) h(y)
$$

and

$$
F(x y)-F\left(x y^{-1}\right)=K(y)+H(x) G(y)
$$

where

$$
\left\{\begin{array}{l}
f(x):=f_{1}(x)+f_{2}(x), p(x):=2 f_{3}(x), q(y):=f_{4}(y)+f_{4}\left(y^{-1}\right) \\
g(x):=f_{5}(x), h(y):=f_{6}(y)+f_{6}\left(y^{-1}\right)
\end{array}\right.
$$

and

$$
\left\{\begin{array}{l}
F(x):=f_{1}(x)-f_{2}(x), K(y):=f_{4}(y)-f_{4}\left(y^{-1}\right), \\
H(x):=f_{5}(x), G(y):=f_{6}(y)-f_{6}\left(y^{-1}\right) .
\end{array}\right.
$$

This paper is organized as follows: In Section 2, we give some terminology and preliminary results which will be used in solving equations (1.3) and (1.4). In Section 3, we present the general solution of the equation (1.3). In Section 4, we solve (1.4). Section 5 contains the general solution of (FE). In Section 6, we determine the general solution of Swiatak's equation; and finally in Section 7, we present the general solution of (SEs).

\section{TERMINOLOGY AND SOME PRELIMINARY RESULTS} if

Let $\mathbf{G}$ be a group and $\mathbf{K}$ be a field. A map $\psi: \mathbf{G} \rightarrow \mathbf{K}$ is called exponential

$$
\psi(x y)=\psi(x) \psi(y) \quad(x, y \in \mathbf{G}) .
$$


We call $\phi: \mathbf{G} \rightarrow \mathbf{K}$ and $A: \mathbf{G} \times \mathbf{G} \rightarrow \mathbf{K}$ additive and biadditive, respectively, if

$$
\phi(x y)=\phi(x)+\phi(y) \quad(x, y \in \mathbf{G}),
$$

and

$$
\begin{array}{r}
A(x y, z)=A(x, z)+A(y, z), \quad A(x, y z)=A(x, y)+A(x, z) \\
(x, y, z \in \mathbf{G}) .
\end{array}
$$

The diagonal of $A$, denoted by $A^{2}$, is defined by

$$
A^{2}(x)=A(x, x) \quad(x \in \mathbf{G}) .
$$

Lemma 2.1. Let $\psi: \mathbf{G} \rightarrow \mathbf{C}$ be exponential, $\psi(x) \not \equiv 0$, and $\psi(x)+\psi(x)^{-1} \not \equiv 2$. Then the general solution $Q, L: \mathbf{G} \rightarrow \mathbf{C}$ of

$$
Q(x y)+Q\left(x y^{-1}\right)=2 Q(x)+2 Q(y)+L(x)\left[\psi(y)+\psi(y)^{-1}-2\right] \quad(x, y \in \mathbf{G}),
$$

where $Q$ satisfies the factorization condition (FC), is given by

$$
\begin{aligned}
& L(x)=\alpha\left[\psi(x)+\psi(x)^{-1}-2\right], \\
& Q(x)=\alpha\left[\psi(x)+\psi(x)^{-1}-2\right]+A^{2}(x) \quad(x \in \mathbf{G}),
\end{aligned}
$$

where $\alpha$ is an arbitrary constant, and $A^{2}$ is the diagonal of an arbitrary biadditive $A$.

Proof. Replacing $y$ by $y^{-1}$ in (2.5) we get

$$
Q(y)=Q\left(y^{-1}\right) .
$$

If we interchange $x$ and $y$ in (2.5), and take the condition (FC) and (2.7) into consideration, we get

$$
L(x)\left[\psi(y)+\psi(y)^{-1}-2\right]=L(y)\left[\psi(x)+\psi(x)^{-1}-2\right] .
$$

Since $\psi(y)+\psi(y)^{-1} \not \equiv 2$, we have

$$
L(x)=\alpha\left[\psi(x)+\psi(x)^{-1}-2\right]
$$

where $\alpha$ is a constant. Substitution of (2.9) into (2.5) yields

$Q(x y)+Q\left(x y^{-1}\right)=2 Q(x)+2 Q(y)+\alpha\left[\psi(x)+\psi(x)^{-1}-2\right]\left[\psi(y)+\psi(y)^{-1}-2\right]$, or

$$
\begin{aligned}
& Q(x y)-\alpha\left[\psi(x y)+\psi(x y)^{-1}-2\right]+Q\left(x y^{-1}\right)-\alpha\left[\psi\left(x y^{-1}\right)+\psi\left(x y^{-1}\right)^{-1}-2\right] \\
& \quad=2\left\{Q(x)-\alpha\left[\psi(x)+\psi(x)^{-1}-2\right]\right\}+2\left\{Q(y)-\alpha\left[\psi(y)+\psi(y)^{-1}-2\right]\right\} .
\end{aligned}
$$

The general solution of this is given by (see [1], Lemma 2)

$$
Q(x)=\alpha\left[\psi(x)+\psi(x)^{-1}-2\right]+A^{2}(x),
$$

where $A^{2}$ is the diagonal of an arbitrary biadditive function $A: \mathbf{G}^{2} \rightarrow \mathbf{C}$. Along with (2.9) we get the necessary form (2.6). The converse is obvious. This completes the proof. $\neq 2$.

We notice that here $\mathbf{C}$ may be replaced by any commutative field $\mathbf{K}$, char $\mathbf{K}$ 
Lemma 2.2. Let $A_{o}^{2}$ be the diagonal of a biadditive function $A_{o}: \mathbf{G}^{2} \rightarrow \mathbf{C}$. Then the general solution $f: \mathbf{G} \rightarrow \mathbf{C}$ of

$$
f(x y)+f\left(x y^{-1}\right)=2 f(x)+2 f(y)+A_{o}^{2}(x) A_{o}^{2}(y) \quad(x, y \in \mathbf{G})
$$

where $f$ satisfies $(\mathrm{FC})$ is given by

$$
f(x)=3 \alpha^{2} \phi(x)^{4}+A^{2}(x), \quad A_{o}^{2}(x)=6 \alpha \phi(x)^{2} \quad(x, y \in \mathbf{G}),
$$

where $\alpha$ is an arbitrary constant, $\phi: \mathbf{G} \rightarrow \mathbf{C}$ an arbitrary additive map and $A^{2}$ the diagonal of an arbitrary biadditive function $A: \mathbf{G}^{2} \rightarrow \mathbf{C}$.

Proof. It is easy to check that $f$ given by (2.11) does satisfy (2.10) and (FC).

In order to prove the converse, we define $F$ by

$$
F(x, t)=f(x t)-f(x)-f(t) .
$$

From (2.10), (FC) and (2.12) we get

$$
\begin{aligned}
& F(x y, t) A+F\left(x y^{-1}, t\right) \\
& =\quad f(x y t)-f(x y)-2 f(t)+f\left(x y^{-1} t\right)-f\left(x y^{-1}\right) \\
& =f(x y t)-f(x y)-2 f(t)-f(x t y)+2 f(x t)+2 f(y)+A_{o}^{2}(x t) A_{o}^{2}(y) \\
& \quad \quad+f(x y)-2 f(x)-2 f(y)-A_{o}^{2}(x) A_{o}^{2}(y) \\
& =2 f(x t)-2 f(x)-2 f(t)+\left[A_{o}^{2}(x t)-A_{o}^{2}(x)\right] A_{o}^{2}(y) \\
& =2 F(x, t)+\left[A_{o}^{2}(x t)-A_{o}^{2}(x)\right] A_{o}^{2}(y) .
\end{aligned}
$$

Since $A_{o}^{2}$ satisfies (FC) and $A_{o}^{2}(x t)-A_{o}^{2}(x)-A_{o}^{2}(t)=A_{o}(x, t)+A_{o}(t, x)$, we have

(2.14) $F(x y, t)+F\left(x y^{-1}, t\right)=2 F(x, t)+\left[A_{o}^{2}(t)+A_{o}(x, t)+A_{o}(t, x)\right] A_{o}^{2}(y)$.

If $A_{o}(x, t)+A_{o}(t, x) \not \equiv 0$, then we choose and fix some $t \in \mathbf{G}$ for which

$$
\phi(x):=A_{o}(x, t)+A_{o}(t, x) \not \equiv 0 .
$$

Since $A_{o}$ is biadditive and $\phi: \mathbf{G} \rightarrow \mathbf{C}$ is additive, applying Lemma 4 in [1] to (2.14), with (2.15), we get

$$
A_{o}^{2}(y)=6 \alpha\left[A_{o}(y, t)+A_{o}(t, y)\right]^{2}=6 \alpha \phi(y)^{2}
$$

for some constant $\alpha$. On the other hand, if $A_{o}(x, t)+A_{o}(t, x) \equiv 0$, then $A_{o}$ is skew-symmetric. In this case $A_{o}^{2}(x)=A_{o}(x, x) \equiv 0$, and (2.16) holds with $\alpha=0$. Thus (2.16) holds in any case.

Substitution of (2.16) into (2.10) yields

$$
f(x y)+f\left(x y^{-1}\right)=2 f(x)+2 f(y)+36 \alpha^{2} \phi(x)^{2} \phi(y)^{2},
$$

that is

$$
\begin{aligned}
f(x y) & -3 \alpha^{2} \phi(x y)^{4}+f\left(x y^{-1}\right)-3 \alpha^{2} \phi\left(x y^{-1}\right)^{4} \\
& =2\left\{f(x)-3 \alpha^{2} \phi(x)^{4}\right\}+2\left\{f(y)-3 \alpha^{2} \phi(y)^{4}\right\} .
\end{aligned}
$$

This yields (see [1], Lemma 2) $f(x)=3 \alpha^{2} \phi(x)^{4}+A^{2}(x)$, where $A^{2}$ is the diagonal of a biadditive function, as asserted in (2.11).

Notice that here $\mathbf{C}$ may be replaced by any commutative field of characteristic different from 2 and 3. 
Lemma 2.3. Let $\mathbf{G}$ be a group and suppose that $f_{1}, f_{2}, f_{3}, f_{4}, f_{5}, f_{6}: \mathbf{G} \rightarrow \mathbf{C}$ satisfy

$$
f_{1}(x y)+f_{2}\left(x y^{-1}\right)=f_{3}(x)+f_{4}(y)+f_{5}(x) f_{6}(y)
$$

and that $f_{1}$ and $f_{2}$ satisfy (FC). If $f_{5}(x)=a_{5}$ (constant), then $f_{3}$ and $f_{4}+a_{5} f_{6}$ satisfy (FC). If $f_{6}(y)=a_{6}$ (constant), then $f_{4}$ and $f_{3}+a_{6} f_{5}$ satisfy (FC). If $f_{5}$ is nonconstant, then $f_{4}$ and $f_{6}$ satisfy (FC). If $f_{6}$ is nonconstant, then $f_{3}$ and $f_{5}$ satisfy $(\mathrm{FC})$.

Proof. It follows from (FC) on $f_{1}$ and $f_{2}$ that

$$
f_{i}(t x y z)=f_{i}(t x(y z))=f_{i}(t(y z) x)=f_{i}((t y) z x)=f_{i}(t y x z)
$$

for $i=1,2$ and for all $t, x, y, z \in \mathbf{G}$. This is a more convenient version of (FC). If $f_{5}(x)=a_{5},(2.17)$ yields

$$
f_{3}(x)=f_{1}\left(x y_{o}\right)+f_{2}\left(x y_{o}^{-1}\right)-f_{4}\left(y_{o}\right)-a_{5} f_{6}\left(y_{o}\right)
$$

and

$$
f_{4}(y)+a_{5} f_{6}(y)=f_{1}\left(x_{o} y\right)+f_{2}\left(x_{o} y^{-1}\right)-f_{3}\left(x_{o}\right)
$$

where $x_{o}$ and $y_{o}$ are fixed elements of $\mathbf{G}$. By applying (2.18) to the above expressions, we see that $f_{3}$ and $f_{4}+a_{5} f_{6}$ satisfy (FC):

$$
\begin{aligned}
& f_{3}(t x y)=f_{1}\left(t x y y_{o}\right)+f_{2}\left(t x y y_{o}^{-1}\right)-f_{4}\left(y_{o}\right)-a_{5} f_{6}\left(y_{o}\right) \\
& \quad=f_{1}\left(t y x y_{o}\right)+f_{2}\left(t y x y_{o}^{-1}\right)-f_{4}\left(y_{o}\right)-a_{5} f_{6}\left(y_{o}\right) \\
& \quad=f_{3}(t y x)
\end{aligned}
$$

and

$$
\begin{aligned}
f_{4}(t x y) & +a_{5} f_{6}(t x y)=f_{1}\left(\left(x_{o} t\right) x y\right)+f_{2}\left(x_{o} y^{-1} x^{-1} t^{-1}\right)-f_{3}\left(x_{o}\right) \\
& =f_{1}\left(x_{o} t y x\right)+f_{2}\left(x_{o} x^{-1} y^{-1} t^{-1}\right)-f_{3}\left(x_{o}\right) \\
& =f_{4}(t y x)+a_{5} f_{6}(t y x) .
\end{aligned}
$$

The case $f_{6}(y)=a_{6}$ is similar.

If $f_{5}(x)$ is nonconstant, then there exist elements $x_{1}, x_{2} \in \mathbf{G}$ such that $f_{5}\left(x_{2}\right)-f_{5}\left(x_{1}\right) \neq 0$. Substituting $x=x_{1}$ and $x=x_{2}$ into (2.17) respectively, we get from the two resulting equations

$$
f_{4}(y)=\frac{f_{5}\left(x_{2}\right)\left[f_{1}\left(x_{1} y\right)+f_{2}\left(x_{1} y^{-1}\right)-f_{3}\left(x_{1}\right)\right]-f_{5}\left(x_{1}\right)\left[f_{1}\left(x_{2} y\right)+f_{2}\left(x_{2} y^{-1}\right)-f_{3}\left(x_{2}\right)\right]}{f_{5}\left(x_{2}\right)-f_{5}\left(x_{1}\right)},
$$

and

$$
f_{6}(y)=\frac{f_{1}\left(x_{2} y\right)+f_{2}\left(x_{2} y^{-1}\right)-f_{3}\left(x_{2}\right)-f_{1}\left(x_{1} y\right)-f_{2}\left(x_{1} y^{-1}\right)+f_{3}\left(x_{1}\right)}{f_{5}\left(x_{2}\right)-f_{5}\left(x_{1}\right)} .
$$

Thus $f_{4}$ and $f_{6}$ inherit (FC) from $f_{1}$ and $f_{2}$. The case $f_{6} \neq$ constant is dealt with in a similar manner. This completes the proof of Lemma 2.3.

Notice that here $\mathbf{C}$ may be replaced by any commutative field.

Lemma 2.4. A function $\phi: \mathbf{G} \rightarrow \mathbf{C}$ satisfies

$$
\phi(x y)-\phi\left(x y^{-1}\right)=2 \phi(y) \quad(x, y \in \mathbf{G})
$$

and

$$
\phi(x y)=\phi(y x) \quad(x, y \in \mathbf{G})
$$

if and only if it is additive. 
Proof. If $\phi$ is additive, it obviously satisfies (2.19) and (2.20). In order to prove the converse, we put $x=e$ (the unit element of $\mathbf{G}$ ) in (2.19) to obtain

$$
\phi(y)=-\phi\left(y^{-1}\right) \text {. }
$$

Interchanging $x$ and $y$ in (2.19), we get

$$
\phi(y x)-\phi\left(y x^{-1}\right)=2 \phi(x) \text {. }
$$

Adding this last equation to (2.19) and using (2.20) and (2.21), we obtain $2 \phi(x y)=2 \phi(x)+2 \phi(y)$ and so $\phi$ is additive.

We notice that here $\mathbf{C}$ may be replaced by any abelian group which is 2 torsion free.

Lemma 2.5. Let $\phi: \mathbf{G} \rightarrow \mathbf{C}$ be additive. Then the general solution $F: \mathbf{G} \rightarrow \mathbf{C}$ of the equation

$$
F(y z)-F\left(y z^{-1}\right)=\phi(z) \quad(y, z \in \mathbf{G})
$$

is given by

$$
F(x)=\frac{1}{2} \phi(x)+\theta(x) \quad(x \in \mathbf{G})
$$

where $\theta: \mathbf{G} \rightarrow \mathbf{C}$ satisfies

$$
\theta\left(x y^{2}\right)=\theta(x) \quad(x, y \in \mathbf{G}) .
$$

Proof. Since $\phi$ is additive it satisfies $\phi(x y)-\phi\left(x y^{-1}\right)=\phi(y)-\phi\left(y^{-1}\right)=2 \phi(y)$. Thus (2.22) can be written as

$$
F(y z)-F\left(y z^{-1}\right)=\frac{1}{2}\left\{\phi(y z)-\phi\left(y z^{-1}\right)\right\}
$$

for all $y, z \in \mathbf{G}$. That is, the map $\theta: \mathbf{G} \rightarrow \mathbf{C}$ defined by

$$
\theta(x):=F(x)-\frac{1}{2} \phi(x)
$$

satisfies

$$
\theta(y z)=\theta\left(y z^{-1}\right) .
$$

From (2.25) we have (2.23), and (2.24) is equivalent to (2.26). This completes the proof. by 2 .

Note that $\mathbf{C}$ may be replaced by any abelian group with unique divisibility

Remark 1. Let $H$ be the subgroup of $\mathbf{G}$ generated by $\mathbf{G}^{2}=\left\{g^{2} \mid g \in \mathbf{G}\right\}$, then (2.24) is equivalent to the statement that $\theta$ is constant on each left coset of $\mathbf{H}$ in $\mathbf{G}$. This is because (2.24) implies $\theta\left(x y_{1}^{2} y_{2}^{2} \cdots y_{n}^{2}\right)=\theta(x)$ by induction, and $\mathbf{H}=\left\{y_{1}^{2} y_{2}^{2} \cdots y_{n}^{2} \mid y_{i} \in \mathbf{G}, n \in \mathscr{N}\right\}$. Here $\mathscr{N}$ denotes the set of natural numbers.

Remark 2. If $\psi_{1}, \psi_{2}, \ldots, \psi_{n}$ are distinct nonzero exponentials, then they are linearly independent. 
Lemma 2.6. Let $f: \mathbf{G} \rightarrow \mathbf{C}$ be a mapping which satisfies (FC) and

$$
f(x y)-f\left(x y^{-1}\right)=f(y)\left\{\psi(x)+\psi(x)^{-1}\right\} \quad(x, y \in \mathbf{G}),
$$

where $\psi: \mathbf{G} \rightarrow \mathbf{C}$ is a nonzero exponential. Then $f$ has one of the following forms:

$$
f(x)=\alpha\left\{\psi(x)-\psi(x)^{-1}\right\} \quad(x \in \mathbf{G}),
$$

or

$$
f(x)=\psi_{o}(x) \phi(x), \quad \psi(x) \equiv \psi(x)^{-1} \equiv \psi_{o}(x) \quad(x \in \mathbf{G}),
$$

where $\phi$ is an arbitrary additive map and $\alpha$ is an arbitrary constant. The converse also holds.

Proof. Setting $x=e$, we obtain

$$
f(y)=-f\left(y^{-1}\right) .
$$

Interchanging $x$ and $y$ in (2.27) and using $f(x y)=f(y x)$ and (2.30), we obtain

$$
f(x y)+f\left(x y^{-1}\right)=f(x)\left[\psi(y)+\psi(y)^{-1}\right] .
$$

The general solution of this equation can be deduced from Lemma 3 in [1] (by setting $g(x)=f(x)$ and $\left.p(y)=\psi(y)+\psi(y)^{-1}\right)$. Hence $f$ necessarily has the form

$$
f(x)=\alpha \psi(x)+\beta \psi(x)^{-1}, \quad \psi(x) \not \equiv \psi(x)^{-1},
$$

or for some nonzero exponential $\psi_{o}$ with $\psi_{o}(y) \equiv \psi_{o}(y)^{-1}$,

$$
f(x)=\psi_{o}(x)[\phi(x)+a], \quad 2 \psi_{o}(y)=\psi(y)+\psi(y)^{-1} .
$$

Since $f$ is odd in $\mathbf{G}$ and $\psi(e)=1, \phi(e)=0$, by putting $x=e$ in (2.32), (2.33) we get $\alpha+\beta=0$ and $a=0$. Thus (2.32) and (2.33) become (2.28) and (2.29), respectively. The converse is straightforward, and this completes the proof of the lemma.

Lemma $2.7[1,5]$. The general solution $f, g: \mathbf{G} \rightarrow \mathbf{C}$ of

$$
f(x y)+f\left(x y^{-1}\right)=2 f(x)+g(y) \quad(x, y \in \mathbf{G})
$$

with $f$ satisfying $(\mathrm{FC})$ is given by

$$
f(x)=A^{2}(x)+\phi(x)+d, \quad g(x)=2 A^{2}(x) \quad(x \in \mathbf{G}),
$$

where $\phi$ is an arbitrary additive map, $A^{2}$ is the diagonal of a symmetric biadditive map and $d$ is an arbitrary constant.

Here again, in Lemmas 2.6 and $2.7, \mathrm{C}$ can be replaced by any quadratically closed commutative field of characteristic different from 2.

\section{Solution OF THE FUNCTIONAL EQUATION (1.3)}

Theorem 3.1. Let $\mathbf{G}$ be a group. The complete list of functions $f, p, q, g, h$ : $\mathbf{G} \rightarrow \mathbf{C}$ which satisfy

$$
f(x y)+f\left(x y^{-1}\right)=p(x)+q(y)+g(x) h(y)
$$


and with $f$ satisfying $(\mathrm{FC})$ is given by

$$
\begin{aligned}
& \left\{\begin{array}{l}
f(x)=A^{2}(x)+\phi(x)+d, \\
p(x)=2 A^{2}(x)+2 \phi(x)-2 b+2 d, \\
q(y)=2 A^{2}(y)-c h(y)+2 b, \\
g(x)=c, \quad h(y) \quad \text { arbitrary, }
\end{array}\right. \\
& \left\{\begin{array}{l}
f(x)=A^{2}(x)+\phi(x)+d, \\
p(x)=2 A^{2}(x)+2 \phi(x)-2 b g(x)-2 a+2 d, \\
q(y)=2 A^{2}(y)+2 a, \quad g(x) \quad \text { arbitrary, } \\
h(y)=2 b, \quad
\end{array}\right. \\
& \left\{\begin{aligned}
f(x)=\gamma\left[\alpha \psi(x)+\beta \psi(x)^{-1}\right]+A^{2}(x)+\phi(x)+d, & \\
p(x)= & 2(\gamma-b)\left[\alpha \psi(x)+\beta \psi(x)^{-1}\right] \\
& \quad+2 A^{2}(x)+2 \phi(x)-2 a+2 b \delta+2 d, \\
q(y)= & \gamma \delta\left[\psi(y)+\psi(y)^{-1}-2\right]+2 A^{2}(y)+2 a, \\
g(x)= & \alpha \psi(x)+\beta \psi(x)^{-1}-\delta, \\
h(y)= & \gamma\left[\psi(y)+\psi(y)^{-1}-2\right]+2 b, \quad \psi(x) \not \equiv \psi(x)^{-1},
\end{aligned}\right.
\end{aligned}
$$

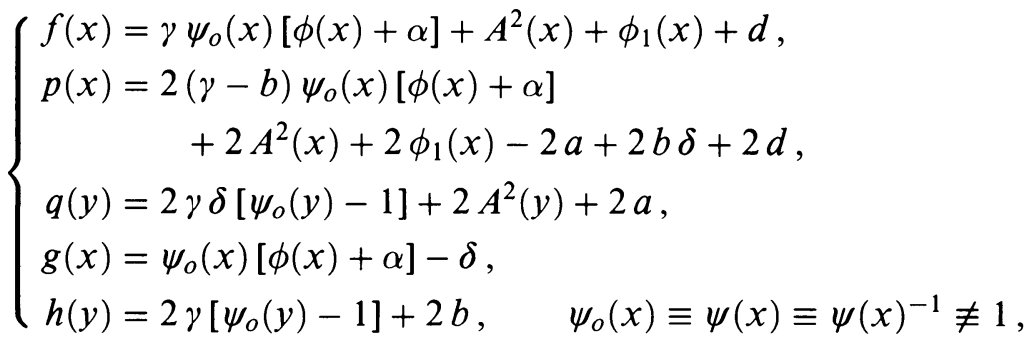

$$
\begin{aligned}
& \left\{\begin{aligned}
f(x)= & \frac{3}{2} \alpha \beta \phi(x)^{4}+\alpha \gamma \phi(x)^{3}-3 \alpha \delta \phi(x)^{2}+A^{2}(x)+\phi_{1}(x)+d, \\
p(x)= & 3 \alpha \beta \phi(x)^{4}+2 \alpha \gamma \phi(x)^{3}-6(\alpha \delta+\beta b) \phi(x)^{2} \\
& \quad+2 A^{2}(x)-2 b \gamma \phi(x)+2 \phi_{1}(x)-2 a+2 b \delta+2 d, \\
q(y)= & 3 \alpha \beta \phi(y)^{4}+2 A^{2}(y)+2 a, \\
g(x)= & 3 \beta \phi(x)^{2}+\gamma \phi(x)-\delta, \\
h(y)= & 6 \alpha \phi(y)^{2}+2 b
\end{aligned}\right.
\end{aligned}
$$

for all $x, y \in \mathbf{G}$. Here $\alpha, \beta, \gamma, \delta, a, b, c, d$ are arbitrary complex constants, $\phi$ and $\phi_{1}$ are arbitrary additive maps, $\psi$ is an abitrary nonzero exponential map, and $A^{2}$ is the diagonal of a biadditive map.

Proof. It is easy to check that all the systems enumerated above satisfy (3.1) with $f$ satisfying (FC).

In order to prove the converse, we first set $y=e$ in (3.1) and get

$$
p(x)=2 f(x)-b_{o} g(x)-a_{o},
$$


and then substituting (3.7) into (3.1), we obtain

$$
f(x y)+f\left(x y^{-1}\right)=2 f(x)+Q(y)+g(x) H(y),
$$

where

(3.9)
$a_{o}:=q(e)$,
$b_{o}:=h(e)$,
$Q(y):=q(y)-a_{o}$,
$H(y):=h(y)-b_{o}$.

Case 1. Suppose $g$ is constant, say $g(x) \equiv c$. Then (3.8) takes the form

$$
f(x y)+f\left(x y^{-1}\right)=2 f(x)+M(y),
$$

where

$$
M(y):=Q(y)+c H(y) .
$$

Hence from Lemma 2.7, we obtain

$$
M(y)=2 A^{2}(y), \quad f(x)=A^{2}(x)+\phi(x)+d,
$$

where $A^{2}$ is the diagonal of a biadditive function, $\phi$ is additive, and $d$ is a constant. From (3.12), (3.11), (3.9) and (3.7) we get solution (3.2).

Case 2. Suppose $h$ is constant, that is $H(y) \equiv 0$. Then (3.7) takes the form

$$
f(x y)+f\left(x y^{-1}\right)=2 f(x)+Q(y) .
$$

Once again, we get from Lemma 2.7

$$
Q(y)=2 A^{2}(y), \quad f(x)=A^{2}(x)+\phi(x)+d .
$$

From (3.14), (3.9), (3.7) and $H(y) \equiv 0$ we get solution (3.3).

Case 3. Suppose $g(x) \not \equiv c$ and $H(y) \not \equiv 0$. Then $h(y) \not \equiv$ constant, and by Lemma 2.3 we conclude that all functions in (3.1) and (3.8) satisfy (FC).

From (3.8) we get

$$
\begin{aligned}
f(x y \cdot z)+f\left(x y \cdot z^{-1}\right) & =2 f(x y)+Q(z)+g(x y) H(z), \\
f\left(x y^{-1} \cdot z\right)+f\left(x y^{-1} \cdot z^{-1}\right) & =2 f\left(x y^{-1}\right)+Q(z)+g\left(x y^{-1}\right) H(z), \\
f(x \cdot y z)+f\left(x \cdot z^{-1} y^{-1}\right) & =2 f(x)+Q(y z)+g(x) H(y z), \\
f\left(x \cdot y z^{-1}\right)+f\left(x \cdot z y^{-1}\right) & =2 f(x)+Q\left(y z^{-1}\right)+g(x) H\left(y z^{-1}\right) .
\end{aligned}
$$

Subtracting the sum of the third and fourth equations from the sum of the first two equations, in view of (3.8) and (FC), we get

$$
\begin{aligned}
& 2 Q(y)+2 g(x) H(y)+\left[g(x y)+g\left(x y^{-1}\right)\right] H(z) \\
& -\left[Q(y z)+Q\left(y z^{-1}\right)-2 Q(z)\right]-g(x)\left[H(y z)+H\left(y z^{-1}\right)\right]=0,
\end{aligned}
$$

that is,

$$
\left\{\begin{aligned}
g(x)[ & \left.H(y z)+H\left(y z^{-1}\right)-2 H(y)\right] \\
& +\left[Q(y z)+Q\left(y z^{-1}\right)-2 Q(y)-2 Q(z)\right] \\
= & {\left[g(x y)+g\left(x y^{-1}\right)\right] H(z) . }
\end{aligned}\right.
$$

Since $g(x) \not \equiv c$, there exist two elements $x_{1}, x_{2} \in \mathbf{G}$ such that $g\left(x_{1}\right) \neq g\left(x_{2}\right)$. Setting $x=x_{1}, x=x_{2}$ in (3.15) respectively we get

$$
\begin{aligned}
g\left(x_{1}\right)[H(y z) & \left.+H\left(y z^{-1}\right)-2 H(y)\right]+\left[Q(y z)+Q\left(y z^{-1}\right)-2 Q(y)-2 Q(z)\right] \\
& =\left[g\left(x_{1} y\right)+g\left(x_{1} y^{-1}\right)\right] H(z),
\end{aligned}
$$




$$
\begin{aligned}
g\left(x_{2}\right)[H(y z) & \left.+H\left(y z^{-1}\right)-2 H(y)\right]+\left[Q(y z)+Q\left(y z^{-1}\right)-2 Q(y)-2 Q(z)\right] \\
& =\left[g\left(x_{2} y\right)+g\left(x_{2} y^{-1}\right)\right] H(z) .
\end{aligned}
$$

From the last two equations we get

$$
H(y z)+H\left(y z^{-1}\right)-2 H(y)=K(y) H(z),
$$

and

$$
Q(y z)+Q\left(y z^{-1}\right)-2 Q(y)-2 Q(z)=L(y) H(z),
$$

where

$$
\left\{\begin{array}{l}
K(y)=\frac{g\left(x_{1} y\right)+g\left(x_{1} y^{-1}\right)-g\left(x_{2} y\right)-g\left(x_{2} y^{-1}\right)}{g\left(x_{1}\right)-g\left(x_{2}\right)}, \\
L(y)=\frac{g\left(x_{1}\right)\left[g\left(x_{2} y\right)+g\left(x_{2} y^{-1}\right)\right]-g\left(x_{2}\right)\left[g\left(x_{1} y\right)+g\left(x_{1} y^{-1}\right)\right]}{g\left(x_{1}\right)-g\left(x_{2}\right)} .
\end{array}\right.
$$

The fact that $f, p, q, g, h$ satisfy the factorization condition (FC) means that the same is true of $Q, H, K, L$. From here on, it is implicitly understood that further functions induced will inherit the factorization condition (FC) without explicit mention.

Substitution of (3.16) and (3.17) into (3.15) yields

$$
g(x) K(y) H(z)+L(y) H(z)=\left[g(x y)+g\left(x y^{-1}\right)\right] H(z) .
$$

Since $H(z) \not \equiv 0$, this gives

$$
g(x y)+g\left(x y^{-1}\right)=g(x) K(y)+L(y) .
$$

From (3.18) with $y=e$ it is easy to note that $K \not \equiv 0$. Further recall that $H \not \equiv 0$. Hence applying the Main Theorem in [1] to (3.16), we obtain

$$
K(x)=\psi(x)+\psi(x)^{-1} \not \equiv 2, \quad H(y)=\gamma\left[\psi(y)+\psi(y)^{-1}-2\right]
$$

or

$$
K(x)=2, \quad H(y)=A_{o}^{2}(y) \not \equiv 0,
$$

where $\gamma \neq 0$ is a constant, $\psi$ a nonzero exponential and $A_{o}^{2}$ the diagonal of a biadditive function.

Subcase 3.1. Suppose $K$ and $H$ are given by (3.20). Then (3.17) yields

$$
Q(x y)+Q\left(x y^{-1}\right)=2 Q(x)+2 Q(y)+\gamma L(x)\left[\psi(y)+\psi(y)^{-1}-2\right] .
$$

This is an equation of the form taken care of in Lemma 2.1, so

$$
\begin{gathered}
L(x)=\delta\left[\psi(x)+\psi(x)^{-1}-2\right], \\
Q(y)=\gamma \delta\left[\psi(y)+\psi(y)^{-1}-2\right]+2 A^{2}(y)
\end{gathered}
$$

where $\delta$ is a constant, and $A^{2}$ is the diagonal of a biadditive map. Substitution of (3.20) and (3.23) into (3.19) yields

$$
g(x y)+g\left(x y^{-1}\right)=g(x)\left[\psi(y)+\psi(y)^{-1}\right]+\delta\left[\psi(y)+\psi(y)^{-1}-2\right],
$$


that is

$$
G(x y)+G\left(x y^{-1}\right)=G(x) P(y), \quad P(x y)+P\left(x y^{-1}\right)=P(x) P(y),
$$

where $G(x):=g(x)+\delta, \quad P(x):=\psi(x)+\psi(x)^{-1}$. By Lemma 3 in [1] and Remark 2, we obtain

$$
g(x)=\alpha \psi(x)+\beta \psi(x)^{-1}-\delta, \quad \psi(x) \not \equiv \psi(x)^{-1}
$$

or

$$
g(x)=\psi_{o}(x)[\phi(x)+\alpha]-\delta, \quad \psi_{o}(x) \equiv \psi(x) \equiv \psi(x)^{-1},
$$

where $\alpha, \beta$ are arbitrary constants, $\phi$ is an arbitrary additive map.

Subcase 3.1.1. Suppose $g$ is given by (3.25). Substitution of (3.20), (3.24) and (3.25) into (3.8) yields

$$
\begin{aligned}
f(x y)+f\left(x y^{-1}\right)= & 2 f(x)+\gamma \delta\left[\psi(y)+\psi(y)^{-1}-2\right] \\
& +2 A^{2}(y)+\gamma\left[\alpha \psi(x)+\beta \psi(x)^{-1}-\delta\right]\left[\psi(y)+\psi(y)^{-1}-2\right],
\end{aligned}
$$

that is

$$
\begin{aligned}
f(x y)-\gamma\left[\alpha \psi(x y)+\beta \psi(x y)^{-1}\right]-A^{2}(x y)+f\left(x y^{-1}\right) \\
-\gamma\left[\alpha \psi\left(x y^{-1}\right)+\beta \psi\left(x y^{-1}\right)^{-1}\right]-A^{2}\left(x y^{-1}\right) \\
=2\left\{f(x)-\gamma\left[\alpha \psi(x)+\beta \psi(x)^{-1}\right]-A^{2}(x)\right\} .
\end{aligned}
$$

This gives (see [1], Lemma 1)

$$
f(x)=\gamma\left[\alpha \psi(x)+\beta \psi(x)^{-1}\right]+A^{2}(x)+\phi(x)+d,
$$

where $d$ is a constant, $\phi$ an additive map. From (3.27), (3.25), (3.7), (3.24), (3.20) and (3.9) we get solution (3.4).

Subcase 3.1.2. Suppose $g$ is given by (3.26). Substitution of (3.20), (3.24) and (3.26) into (3.8) yields

$$
\begin{aligned}
f(x y)+f\left(x y^{-1}\right)= & 2 f(x)+2 \gamma \delta\left[\psi_{o}(y)-1\right]+2 A^{2}(y) \\
& +2 \gamma\left[\psi_{o}(x)\{\phi(x)+\alpha\}-\delta\right]\left[\psi_{o}(y)-1\right],
\end{aligned}
$$

that is,

$$
\begin{aligned}
f(x y) & -\gamma \psi_{o}(x y)[\phi(x y)+\alpha]-A^{2}(x y)+f\left(x y^{-1}\right) \\
& -\gamma \psi_{o}\left(x y^{-1}\right)\left[\phi\left(x y^{-1}\right)+\alpha\right]-A^{2}\left(x y^{-1}\right) \\
= & 2\left\{f(x)-\gamma \psi_{o}(x)[\phi(x)+\alpha]-A^{2}(x)\right\} .
\end{aligned}
$$

This gives (see [1], Lemma 1) for some additive $\phi_{1}$

$$
f(x)=\gamma \psi_{o}(x)[\phi(x)+\alpha]+A^{2}(x)+\phi_{1}(x)+d .
$$

From (3.28), (3.26), (3.7), (3.24), (3.20) and (3.9) we get solution (3.5).

Subcase 3.2. Suppose $K$ and $H$ are given by (3.21). Substitution of (3.21) into (3.17) yields

$$
Q(x y)+Q\left(x y^{-1}\right)=2 Q(x)+2 Q(y)+L(x) A_{o}^{2}(y) .
$$


Interchanging in (3.29) $y$ and $y^{-1}$, since $A_{o}^{2}(y)=A_{o}^{2}\left(y^{-1}\right)$, we have $Q(y)=$ $Q\left(y^{-1}\right)$. Thus $Q(x y)+Q\left(x y^{-1}\right)=Q(y x)+Q\left(y x^{-1}\right)$ follows under (FC), and from (3.29) we get

$$
L(x) A_{o}^{2}(y)=L(y) A_{o}^{2}(x) .
$$

By $(3.21) A_{o}^{2}(y) \not \equiv 0$ and thus we have

$$
L(y)=c A_{o}^{2}(y) .
$$

Hence the equation (3.29) becomes

$$
Q(x y)+Q\left(x y^{-1}\right)=2 Q(x)+2 Q(y)+c A_{o}^{2}(x) A_{o}^{2}(y) .
$$

Subcase 3.2.1. If $c \neq 0$, then by Lemma 2.2 we get $Q(y)=3 \beta^{2} c^{-1} \phi(y)^{4}+$ $c^{-1} A_{1}^{2}(y)$ and $A_{o}^{2}(x)=6 \beta c^{-1} \phi(x)^{2}$. Defining $\alpha:=\beta c^{-1}$ and $A(x):=$ $(2 c)^{-1} A_{1}(x)$, we have

$$
A_{o}^{2}(x)=6 \alpha \phi(x)^{2}
$$

and

$$
Q(y)=3 \alpha \beta \phi(y)^{4}+2 A^{2}(y),
$$

where $\alpha \neq 0$ is a constant. Substitution of (3.21), (3.30) and (3.32) into (3.19) yields (remembering that $\alpha c=\beta$ )

$$
g(x y)+g\left(x y^{-1}\right)=2 g(x)+6 \beta \phi(y)^{2},
$$

or

$$
g(x y)-3 \beta \phi(x y)^{2}+g\left(x y^{-1}\right)-3 \beta \phi\left(x y^{-1}\right)^{2}=2\left\{g(x)-3 \beta \phi(x)^{2}\right\} .
$$

This gives (see [1], Lemma 1)

$$
g(x)=3 \beta \phi(x)^{2}+\phi_{o}(x)-\delta,
$$

where $\delta$ is an arbitrary constant, $\phi_{o}$ an additive map.

Substitution of (3.21), (3.32), (3.33) and (3.34) into (3.8) yields

$$
\begin{aligned}
f(x y)+f\left(x y^{-1}\right)= & 2 f(x)+3 \alpha \beta \phi(y)^{4}+2 A^{2}(y) \\
& +6 \alpha\left\{3 \beta \phi(x)^{2}+\phi_{o}(x)-\delta\right\} \phi(y)^{2},
\end{aligned}
$$

or

$$
\begin{aligned}
f(x y)- & \frac{3}{2} \alpha \beta \phi(x y)^{4}+3 \alpha \delta \phi(x y)^{2}-A^{2}(x y) \\
& +f\left(x y^{-1}\right)-\frac{3}{2} \alpha \beta \phi\left(x y^{-1}\right)^{4}+3 \alpha \delta \phi\left(x y^{-1}\right)^{2}-A^{2}\left(x y^{-1}\right) \\
= & 2\left\{f(x)-\frac{3}{2} \alpha \beta \phi(x)^{4}+3 \alpha \delta \phi(x)^{2}-A^{2}(x)\right\}+6 \alpha \phi_{o}(x) \phi(y)^{2} .
\end{aligned}
$$

This gives (see [1], Lemma 4, and recall $\alpha \neq 0$ )

$$
f(x)=\frac{3}{2} \alpha \beta \phi(x)^{4}+\alpha \gamma \phi(x)^{3}-3 \alpha \delta \phi(x)^{2}+A^{2}(x)+\phi_{1}(x)+d,
$$

$$
\phi_{o}(x)=\gamma \phi(x),
$$

where $\gamma, \delta$ are constants, $\phi_{1}$ is additive. From (3.34), (3.35), (3.36), (3.33), (3.32), (3.21), (3.7) and (3.9) we get solution (3.6). 
Subcase 3.2.2. Finally, suppose $c=0$ in (3.30). Then (3.31) yields

$$
Q(y)=2 A^{2}(y),
$$

which is a special case of (3.33) with $\beta=0$. Substituting (3.21) along with $L=0$ (from (3.30)) into (3.19), we obtain

$$
g(x y)+g\left(x y^{-1}\right)=2 g(x) .
$$

Hence (from [1], Lemma 1 again) we have

$$
g(x)=\phi(x)-\delta
$$

for arbitrary constant $\delta$ and arbitrary additive $\phi$. (Cf. (3.34) with $\beta=0$.)

Substituting (3.21), (3.37) and (3.38) into (3.8), we get

$$
f(x y)+f\left(x y^{-1}\right)=2 f(x)+2 A^{2}(y)+\{\phi(x)-\delta\} A_{o}^{2}(y),
$$

or

$\left\{f(x y)-A^{2}(x y)\right\}+\left\{f\left(x y^{-1}\right)-A^{2}\left(x y^{-1}\right)\right\}=2\left\{f(x)-A^{2}(x)\right\}+\{\phi(x)-\delta\} A_{o}^{2}(y)$.

Now Lemma 4 in [1] yields (recalling $\phi \not \equiv 0$, since $g$ is nonconstant)

$$
f(x)=\alpha \phi(x)^{3}-3 \alpha \delta \phi(x)^{2}+A^{2}(x)+\phi_{1}(x)+d, \quad A_{o}^{2}(y)=6 \alpha \phi(y)^{2},
$$

where $\alpha$ and $d$ are arbitrary constants, and $\phi_{1}$ is an arbitrary additive map. From (3.39), (3.38), (3.37), (3.21), (3.7) and (3.9), we get a special case of solution (3.6), in which $\beta=0$ and $\gamma=1$.

There are no cases left, so the proof is complete.

\section{Solution of the functional equation (1.4)}

Theorem 4.1. Let $\mathbf{G}$ be a group. The maps $F, G, H, K: \mathbf{G} \rightarrow \mathbf{C}$ satisfy

$$
F(x y)-F\left(x y^{-1}\right)=K(y)+H(x) G(y)
$$$$
(t, x, y \in \mathbf{G})
$$

and with $F$ satisfying (FC), if and only if they have one of the forms

$$
\begin{gathered}
\left\{\begin{array}{c}
F(x)=\frac{1}{2} \phi(x)+\theta(x), \\
K(y)=\phi(y)-a G(y), \\
H(x)=a \\
G \text { arbitrary, }
\end{array}\right. \\
\left\{\begin{array}{c}
F(x)=\frac{1}{2} \phi(x)+\theta(x), \\
K(y)=\phi(y) \\
H \quad \text { arbitrary } \\
G(y)=0
\end{array}\right.
\end{gathered}
$$

$$
\left\{\begin{array}{l}
F(x)=d\left[a \psi(x)-b \psi(x)^{-1}\right]+\frac{1}{2} \phi(x)+\theta(x), \\
K(y)=-d c\left[\psi(y)-\psi(y)^{-1}\right]+\phi(y), \\
H(x)=a \psi(x)+b \psi(x)^{-1}+c, \\
G(y)=d\left[\psi(y)-\psi(y)^{-1}\right], \quad \psi(y) \not \equiv \psi(y)^{-1},
\end{array}\right.
$$




$$
\begin{aligned}
& \left\{\begin{array}{l}
F(x)=\frac{1}{2} \phi_{1}(x)+\frac{1}{2} \psi_{o}(x) \phi_{2}(x)\left[\frac{c}{2} \phi_{2}(x)+a\right]+\theta(x), \\
K(y)=\phi_{1}(y)-b \psi_{o}(y) \phi_{2}(y), \\
H(x)=\psi_{o}(x)\left[c \phi_{2}(x)+a\right]+b, \\
G(y)=\psi_{o}(y) \phi_{2}(y), \quad \psi_{o}(y) \equiv \psi(y) \equiv \psi(y)^{-1} \not \equiv 1,
\end{array}\right. \\
& \left\{\begin{array}{l}
F(x)=\frac{a}{2} \phi_{1}(x)^{3}+\frac{b}{4} \phi_{1}(x)^{2}+\frac{c}{2} \phi_{1}(x)+\frac{1}{2} \phi_{2}(x)+\theta(x), \\
K(y)=a \phi_{1}(y)^{3}+\phi_{2}(y), \\
H(x)=3 a \phi_{1}(x)^{2}+b \phi_{1}(x)+c, \\
G(y)=\phi_{1}(y), \quad \phi_{1} \not \equiv 0
\end{array}\right.
\end{aligned}
$$

for all $x, y \in \mathbf{G}$. Here $a, b, c, d$ are arbitrary constants in $\mathbf{C}, \phi, \phi_{1}, \phi_{2}$ are additive, $\psi$ is a nonzero exponential and $\theta: \mathbf{G} \rightarrow \mathbf{C}$ is any function satisfying (FC) and

$$
\theta\left(x y^{2}\right)=\theta(x), \quad(x, y \in \mathbf{G}) .
$$

Proof. From (1.4) it follows that

$$
\begin{aligned}
F(x y z)-F\left(x y z^{-1}\right) & =K(z)+H(x y) G(z), \\
F\left(x y^{-1} z\right)-F\left(x y^{-1} z^{-1}\right) & =K(z)+H\left(x y^{-1}\right) G(z), \\
F(x y z)-F\left(x z^{-1} y^{-1}\right) & =K(y z)+H(x) G(y z), \\
F\left(x y z^{-1}\right)-F\left(x z y^{-1}\right) & =K\left(y z^{-1}\right)+H(x) G\left(y z^{-1}\right),
\end{aligned}
$$

all hold. Subtracting the sum of the first, second, and fourth equations from the third equation, and using (FC), we obtain

$G(z)\left[H(x y)+H\left(x y^{-1}\right)\right]+2 K(z)-K(y z)+K\left(y z^{-1}\right)=H(x)\left[G(y z)-G\left(y z^{-1}\right)\right]$.

We shall consider several cases.

Case 1. Suppose $H$ is constant, say $H(x) \equiv a \in \mathbf{C}$. Then (4.7) shows that the map $\phi$ defined by

$$
\phi(x):=a G(x)+K(x),
$$

satisfies

$$
2 \phi(z)-\phi(y z)+\phi\left(y z^{-1}\right)=0 .
$$

Thus by Lemmas 2.3 and $2.4, \phi$ is additive, and the general solution of (4.7) is therefore (cf. (4.8)) given by

$$
H(x)=a, \quad G \text { arbitrary, } \quad K(x)=\phi(x)-a G(x) .
$$

Letting these into (1.4), we have

$$
F(x y)-F\left(x y^{-1}\right)=\phi(y) .
$$

Thus, by Lemma 2.5, we have solution (4.1).

Case 2. Suppose $H$ is nonconstant. Now based on whether $G$ is identically zero or not, we have two subcases. 
Subcase 2.1. Suppose $G \equiv 0$. Then (4.7) yields

$$
K(y z)-K\left(y z^{-1}\right)=2 K(z) .
$$

Applying Lemmas 2.3 and 2.4 again, we find that $K$ is additive, say $K=\phi$. Since $G=0, H$ is arbitrary and (1.4) reduces to (4.9) again. This brings us to the solution (4.2).

Subcase 2.2. Suppose $G \not \equiv 0$. Putting $z=z_{1}$ such that $G\left(z_{1}\right) \neq 0$ in (4.7), we obtain

$H(x y)+H\left(x y^{-1}\right)=H(x)\left[\frac{G\left(y z_{1}\right)-G\left(y z_{1}^{-1}\right)}{G\left(z_{1}\right)}\right]+\frac{K\left(y z_{1}\right)-K\left(y z_{1}^{-1}\right)-2 K\left(z_{1}\right)}{G\left(z_{1}\right)}$,

which is an equation of the form of (1.3), with $p \equiv 0$ and $f=g=H$. Examining the forms of solutions provided by Theorem 3.1, we see first of all that (3.2) can be eliminated, since $H$ is presently nonconstant, while $g$ is constant in (3.2). From the form of $f$ in (3.3) or the form of $g$ in (3.6), we have the possibility that

$$
H(x)=A^{2}(x)+\phi(x)+c,
$$

where $\phi: \mathbf{G} \rightarrow \mathbf{C}$ is additive, $c$ is a constant, and $A^{2}$ is the diagonal of a biadditive $A: \mathbf{G}^{2} \rightarrow \mathbf{C}$. Finally, from the forms of $g$ in (3.4) and (3.5), we get, respectively, the possibilities

$$
\begin{aligned}
& H(x)=a \psi(x)+b \psi(x)^{-1}+c, \quad \psi(x) \not \equiv \psi(x)^{-1}, \\
& H(x)=\psi(x)[\phi(x)+a]+b, \quad \psi(x) \equiv \psi(x)^{-1} \not \equiv 1,
\end{aligned}
$$

for some constants $a, b, c$, additive $\phi: \mathbf{G} \rightarrow \mathbf{C}$, and nonzero exponential $\psi$.

Now we consider the three possibilities (4.10), (4.11), (4.12) for $H$ separately.

Subcase 2.2.1. Suppose $H$ is given by (4.10). Then inserting (4.10) into (4.7), we get

$$
\begin{aligned}
2 A^{2}(y) G(z) & +2 K(z)-K(y z)+K\left(y z^{-1}\right) \\
& =\left[A^{2}(x)+\phi(x)+c\right]\left[G(y z)-G\left(y z^{-1}\right)-2 G(z)\right] .
\end{aligned}
$$

Since $H$ is nonconstant, it is evident that $A^{2}+\phi$ is also nonconstant, and we conclude from (4.13) that

$$
2 A^{2}(y) G(z)+2 K(z)-K(y z)+K\left(y z^{-1}\right)=0
$$

and that

$$
G(y z)-G\left(y z^{-1}\right)-2 G(z)=0 .
$$

From the last equation and Lemmas 2.3 and 2.4, we obtain

$$
G=\phi_{1}
$$

where $\phi_{1}: \mathbf{G} \rightarrow \mathbf{C}$ is additive. Substituting this into (4.14), we find that

$$
K(y z)-K\left(y z^{-1}\right)=2 K(z)+2 A^{2}(y) \phi_{1}(z) \text {. }
$$

Interchanging $z$ and $z^{-1}$ in (4.16) we see that $K$ is odd, that is, $K\left(z^{-1}\right)=$ $-K(z)$. Since $K(y z)=K(z y)$ by Lemma 2.3, we rewrite (4.16) as

$$
K(z y)+K\left(z y^{-1}\right)-2 K(z)=2 \phi_{1}(z) A^{2}(y) .
$$


Hence we obtain (see [1], Lemma 4)

$$
K(x)=a \phi_{1}(x)^{3}+\phi_{2}(x), \quad A^{2}(y)=3 a \phi_{1}(y)^{2},
$$

where $\phi_{2}: \mathbf{G} \rightarrow \mathbf{C}$ is additive and $a \in \mathbf{C}$ is an arbitrary constant. Notice that to arrive at $(4.17)$ we have used the facts that $\phi_{1}(=G)$ is not identically zero and $K$ is odd.

Now with (4.17), (4.10) becomes

$$
H(y)=3 a \phi_{1}(y)^{2}+\phi(y)+c .
$$

Substituting (4.18), (4.17), (4.15) back into (1.4), we obtain

$$
F(x y)-F\left(x y^{-1}\right)=a \phi_{1}(y)^{3}+3 a \phi_{1}(x)^{2} \phi_{1}(y)+\phi_{2}(y)+[\phi(x)+c] \phi_{1}(y) .
$$

Next, we define $F_{o}: \mathbf{G} \rightarrow \mathbf{C}$ and $\theta: \mathbf{G} \rightarrow \mathbf{C}$ by

$$
\left\{\begin{aligned}
F_{o}(x) & :=\frac{a}{2} \phi_{1}(x)^{3}+\frac{1}{2} \phi_{2}(x)+\frac{1}{2}\left[\frac{1}{2} \phi(x)+c\right] \phi_{1}(x), \\
\theta(x) & :=F(x)-F_{o}(x) .
\end{aligned}\right.
$$

Calculating $F_{o}(x y)-F_{o}\left(x y^{-1}\right)$ by using the fact that $\phi, \phi_{1}, \phi_{2}$ are additive, we get

$$
\begin{aligned}
F_{o}(x y)-F_{o}\left(x y^{-1}\right)= & a \phi_{1}(y)^{3}+3 a \phi_{1}(x)^{2} \phi_{1}(y) \\
& +\phi_{2}(y)+\frac{1}{2}\left[\phi(x) \phi_{1}(y)+\phi(y) \phi_{1}(x)\right]+c \phi_{1}(y) .
\end{aligned}
$$

Subtracting this from (4.19), we obtain

$$
\theta(x y)-\theta\left(x y^{-1}\right)=\frac{1}{2}\left[\phi(x) \phi_{1}(y)-\phi(y) \phi_{1}(x)\right] .
$$

Since the right side of the last equation is skew-symmetric in $x$ and $y$, the left side must be also skew-symmetric. That is,

$$
\theta(x y)-\theta\left(x y^{-1}\right)=-\left[\theta(y x)-\theta\left(y x^{-1}\right)\right], \quad x, y \in \mathbf{G} .
$$

Since $\theta$ inherits (FC) from $F$, we obtain $2 \theta(x y)=\theta\left(x y^{-1}\right)+\theta\left(y x^{-1}\right)$. With $x=s y \quad(s, y \in \mathbf{G})$, this yields $2 \theta\left(s y^{2}\right)=\theta(s)+\theta\left(s^{-1}\right)$. With $y=e$, the last equation implies $\theta(s)=\theta\left(s^{-1}\right)$, so that $\theta$ satisfies

$$
\theta\left(s y^{2}\right)=\theta(s), \quad s, y \in \mathbf{G} .
$$

This is equivalent to $\theta(x y)-\theta\left(x y^{-1}\right)=0$, and so (4.21) shows that

$$
\phi(x) \phi_{1}(y)=\phi(y) \phi_{1}(x), \quad x, y \in \mathbf{G} .
$$

Choosing $y_{o}$ so that $\phi_{1}\left(y_{o}\right) \neq 0$, we deduce that

$$
\phi(x)=b \phi_{1}(x), \quad x \in \mathbf{G},
$$

for some constant $b \in \mathbf{C}$. Thus, by (4.20), (4.22), (4.17), (4.18), (4.15), we have solution (4.5).

Subcase 2.2.2. Suppose $H$ is given by (4.11). In this case

$$
H(x y)+H\left(x y^{-1}\right)=\left[a \psi(x)+b \psi(x)^{-1}\right]\left[\psi(y)+\psi(y)^{-1}\right]+2 c .
$$


With this, (4.7) can be expressed as

(4.23)

$$
\left\{\begin{aligned}
{[a \psi(x)} & \left.+b \psi(x)^{-1}\right]\left\{\left[\psi(y)+\psi(y)^{-1}\right] G(z)-G(y z)+G\left(y z^{-1}\right)\right\} \\
& =K(y z)-K\left(y z^{-1}\right)-2 K(z)+c\left[G(y z)-G\left(y z^{-1}\right)-2 G(z)\right]
\end{aligned}\right.
$$

Since $H$ is nonconstant, (4.11) shows that also $a \psi(x)+b \psi(x)^{-1}$ is nonconstant, hence both sides of (4.23) must be zero. That is,

$$
G(y z)-G\left(y z^{-1}\right)=\left[\psi(y)+\psi(y)^{-1}\right] G(z),
$$

and

$$
\phi(y z)-\phi\left(y z^{-1}\right)-2 \phi(z)=0,
$$

where $\phi: \mathbf{G} \rightarrow \mathbf{C}$ is defined by

$$
\phi(x):=K(x)+c G(x) .
$$

From Lemmas 2.3 and 2.6, we obtain the solution $G: \mathbf{G} \rightarrow \mathbf{C}$ of (4.24) as

$$
G(x)=d\left[\psi(x)-\psi(x)^{-1}\right],
$$

for some constant $d \in \mathbf{C}$. Also, by Lemmas 2.3 and 2.4, (4.25) implies that $\phi$ is a additive, and (4.26) yields

$$
K(x)=\phi(x)-c d\left[\psi(x)-\psi(x)^{-1}\right] .
$$

Substituting now (4.11), (4.28) and (4.27) into (1.4), we obtain after simplification

$$
F(x y)-F\left(x y^{-1}\right)=\phi(y)+\left[a \psi(x)+b \psi(x)^{-1}\right] d\left[\psi(y)-\psi(y)^{-1}\right] .
$$

Furthermore, defining $F_{o}: \mathbf{G} \rightarrow \mathbf{C}$ by

$$
F_{o}(x):=\frac{1}{2} \phi(x)+d\left[a \psi(x)-b \psi(x)^{-1}\right], \quad x \in \mathbf{G},
$$

we calculate that

(4.31) $\quad F_{o}(x y)-F_{o}\left(x y^{-1}\right)=\phi(y)+d\left[a \psi(x)+b \psi(x)^{-1}\right]\left[\psi(y)-\psi(y)^{-1}\right]$.

Therefore, by (4.29) and (4.31), the maps $\theta: \mathbf{G} \rightarrow \mathbf{C}$ defined by

$$
\theta(x):=F(x)-F_{o}(x),
$$

satisfies again $\theta(x y)-\theta\left(x y^{-1}\right)=0$, that is, (4.6). It inherits (FC) from $F$. Finally, by (4.32), (4.30), (4.28), (4.11), and (4.27), we have solution (4.3) in this case.

Subcase 2.2.3. Finally, we consider the case when $H$ is given by (4.12). It is easy to see that

$$
H(x y)+H\left(x y^{-1}\right)=2 \psi(x) \psi(y)[\phi(x)+a]+2 b,
$$

and (4.7) becomes

$$
\begin{gathered}
\psi(x)[\phi(x)+a]\left\{2 \psi(y) G(z)-G(y z)+G\left(y z^{-1}\right)\right\} \\
=\phi_{1}(y z)-\phi_{1}\left(y z^{-1}\right)-2 A \phi_{1}(z),
\end{gathered}
$$

where $\phi_{1}: \mathbf{G} \rightarrow \mathbf{C}$ is defined by

$$
\phi_{1}(x):=K(x)+b G(x) .
$$


As before, (4.33) implies that both sides must be zero, since $H$ and (by (4.12)) also $\psi[\phi+a]$ must be nonconstant. Thus (4.33) yields

$$
G(y z)-G\left(y z^{-1}\right)=2 \psi(y) G(z), \quad y, z \in \mathbf{G},
$$

and

$$
\phi_{1}(y z)-\phi_{1}\left(y z^{-1}\right)=2 \phi_{1}(z), \quad y, z \in \mathbf{G},
$$

that is, $\phi_{1}$ is additive. Since $\psi(x) \equiv \psi(x)^{-1}$, applying Lemma 2.6 to (4.35), we obtain

$$
G(x)=\psi(x) \phi_{2}(x),
$$

for some additive $\phi_{2}: \mathbf{G} \rightarrow \mathbf{C}$. Then (4.34) yields

$$
K(x)=\phi_{1}(x)-b \psi(x) \phi_{2}(x) .
$$

Substituting (4.37), (4.12) and (4.36) back into (1.4), we have in this case

$$
F(x y)-F\left(x y^{-1}\right)=\phi_{1}(y)+\psi(x) \psi(y)[\phi(x)+a] \phi_{2}(y) .
$$

On the other hand, defining $F_{o}: \mathbf{G} \rightarrow \mathbf{C}$ by

$$
F_{o}(x):=\frac{1}{2} \phi_{1}(x)+\frac{1}{2} \psi(x) \phi_{2}(x)\left[\frac{1}{2} \phi(x)+a\right],
$$

we calculate that

(4.40)

$$
F_{o}(x y)-F_{o}\left(x y^{-1}\right)=\phi_{1}(y)+\psi(x) \psi(y)\left\{\phi_{2}(y)\left[\frac{1}{2} \phi(x)+a\right]+\frac{1}{2} \phi_{2}(x) \phi(y)\right\} \text {. }
$$

Subtracting (4.40) from (4.38), we find that the map $\theta: \mathbf{G} \rightarrow \mathbf{C}$ defined by

$$
\theta(x):=F(x)-F_{o}(x)
$$

satisfies

$$
\theta(x y)-\theta\left(x y^{-1}\right)=\frac{1}{2} \psi(x) \psi(y)\left[\phi_{2}(y) \psi(x)-\phi_{2}(x) \phi(y)\right] .
$$

Interchanging $x$ and $y$, we see that

$$
\theta(x y)-\theta\left(x y^{-1}\right)=-\left[\theta(y x)-\theta\left(y x^{-1}\right)\right] .
$$

As in the case 2.2.1, this leads to the conclusions that $\theta$ satisfies (4.6) and inherits (FC) from $F$ and (since $\psi \phi_{2}=G \not \equiv 0$ ) that

$$
\phi(x)=c \phi_{2}(x), \quad x \in \mathbf{G},
$$

for some $c \in \mathbf{C}$. Now, by (4.41), (4.39), (4.42), (4.37), (4.12) and (4.36), we have solution (4.4). As all systems satisfy (1.4) with $F$ satisfying (FC), the proof is completed.

Remark 3. A special case of Theorem 4.1 in which $K=F$ is proved in [1].

Remark 4. Let $\mathbf{S}$ be the normal subgroup generated by $\mathbf{G}^{2}=\left\{g^{2} \mid g \in \mathbf{G}\right\}$. Then (4.6) along with (FC) is equivalent to the statement that $\theta$ is constant on each coset of $\mathbf{S}$ in group $\mathbf{G}$. 


\section{Solutions of THE FUnCtional EQUATION (FE)}

Using (1.5), (1.6), Theorem 3.1 and Theorem 4.1, the general solution of (FE) with (FC) can be displayed.

Theorem 5.1. Let $\mathbf{G}$ be a group. The complete list of functions $f_{1}, f_{2}, f_{3}, f_{4}$, $f_{5}, f_{6}: \mathbf{G} \rightarrow \mathbf{C}$ which satisfy

$$
f_{1}(x y)+f_{2}\left(x y^{-1}\right)=f_{3}(x)+f_{4}(y)+f_{5}(x) f_{6}(y)
$$

along with the factorization condition (FC) on $f_{1}$ and $f_{2}$ is the following:

$$
\left\{\begin{array}{l}
f_{1}(x)=\frac{1}{2} A^{2}(x)+\frac{1}{2} \phi_{2}(x)+\theta(x), \\
f_{2}(x)=\frac{1}{2} A^{2}(x)+\phi_{1}(x)-\frac{1}{2} \phi_{2}(x)-\theta(x)+d, \\
f_{3}(x)=A^{2}(x)+\phi_{1}(x)-b+d \\
f_{4}(y)=A^{2}(y)-\phi_{1}(y)+\phi_{2}(y)-c f_{6}(y)+b, \\
f_{5}(x)=c \\
f_{6}(y) \text { arbitrary }
\end{array}\right.
$$

$$
\left\{\begin{array}{l}
f_{1}(x)=\frac{1}{2} A^{2}(x)+\frac{1}{2} \phi_{2}(x)+\theta(x), \\
f_{2}(x)=\frac{1}{2} A^{2}(x)+\phi_{1}(x)-\frac{1}{2} \phi_{2}(x)-\theta(x)+d, \\
f_{3}(x)=A^{2}(x)+\phi_{1}(x)-b f_{5}(x)-a+d, \\
f_{4}(y)=A^{2}(y)-\phi_{1}(y)+\phi_{2}(y)+a, \\
f_{5}(x) \text { arbitrary } \\
f_{6}(y)=b
\end{array}\right.
$$

$$
\left\{\begin{aligned}
& f_{1}(x)=\gamma\left[\alpha \psi(x)+\beta \psi(x)^{-1}\right]+\epsilon\left[\alpha \psi(x)-\beta \psi(x)^{-1}\right] \\
&+\frac{1}{2} A^{2}(x)+\frac{1}{2} \phi_{1}(x)+\theta(x), \\
& f_{2}(x)=\gamma\left[\alpha \psi(x)+\beta \psi(x)^{-1}\right]-\epsilon\left[\alpha \psi(x)-\beta \psi(x)^{-1}\right] \\
& \quad+\frac{1}{2} A^{2}(x)-\frac{1}{2} \phi_{1}(x)+\phi(x)-\theta(x)+d, \\
& f_{3}(x)= b\left[\alpha \psi(x)+\beta \psi(x)^{-1}\right]+A^{2}(x)+\phi(x)-a-b \delta+d, \\
& f_{4}(y)= \delta \gamma\left[\psi(y)+\psi(y)^{-1}\right]+\delta \epsilon\left[\psi(y)-\psi(y)^{-1}\right] \\
& \quad+A^{2}(y)+\phi_{1}(y)-\phi(y)+a, \\
& f_{5}(x)=\alpha \psi(x)+\beta \psi(x)^{-1}-\delta, \\
& f_{6}(y)=\gamma\left[\psi(y)+\psi(y)^{-1}\right]+\epsilon\left[\psi(y)-\psi(y)^{-1}\right]-b, \\
& \psi(x) \not \equiv \psi(x)^{-1},
\end{aligned}\right.
$$




$$
\left\{\begin{aligned}
f_{1}(x)= & \frac{\gamma}{2} \psi_{o}(x)[c \phi(x)+\alpha]+\frac{1}{2} \psi_{o}(x) \phi(x)\left[\frac{c}{2} \phi(x)+\alpha\right] \\
& \quad+\frac{1}{2} A^{2}(x)+\frac{1}{2} \phi_{2}(x)+\theta(x), \\
f_{2}(x)= & \frac{\gamma}{2} \psi_{o}(x)[c \phi(x)+\alpha]-\frac{1}{2} \psi_{o}(x) \phi(x)\left[\frac{c}{2} \phi(x)+\alpha\right] \\
& \quad+\frac{1}{2} A^{2}(x)+\phi_{1}(x)-\frac{1}{2} \phi_{2}(x)-\theta(x)+d, \\
f_{3}(x)= & b \psi_{o}(x)[c \phi(x)+\alpha]+A^{2}(x)+\phi_{1}(x)-a-b \delta+d, \\
f_{4}(y)= & \delta \psi_{o}(y)[\phi(y)+\gamma]+A^{2}(y)-\phi_{1}(y)+\phi_{2}(y)+a, \\
f_{5}(x)= & \psi_{o}(x)[c \phi(x)+\alpha]-\delta, \\
f_{6}(y)= & \psi_{o}(y)[\phi(y)+\gamma]-b, \quad \psi_{o}(y) \equiv \psi(y) \equiv \psi(y)^{-1} \not \equiv 1,
\end{aligned}\right.
$$

$$
\begin{aligned}
& f_{1}(x)=3 \alpha \beta \phi(x)^{4}+2(\alpha \gamma+\beta \epsilon) \phi(x)^{3}+(3 \alpha \delta+\gamma \epsilon) \phi(x)^{2} \\
& +\frac{1}{2} A^{2}(x)+\delta \epsilon \phi(x)+\frac{1}{2} \phi_{2}(x)+\theta(x) \text {, } \\
& f_{2}(x)=3 \alpha \beta \phi(x)^{4}+2(\alpha \gamma-\beta \epsilon) \phi(x)^{3}+(3 \alpha \delta-\gamma \epsilon) \phi(x)^{2} \\
& +\frac{1}{2} A^{2}(x)-\delta \epsilon \phi(x)+\phi_{1}(x)-\frac{1}{2} \phi_{2}(x)-\theta(x)+d, \\
& f_{3}(x)=6 \alpha \beta \phi(x)^{4}+4 \alpha \gamma \phi(x)^{3}+6(\alpha \delta-\beta b) \phi(x)^{2} \\
& +A^{2}(x)-2 b \gamma \phi(x)+\phi_{1}(x)-a-b \delta+d, \\
& f_{4}(y)=6 \alpha \beta \phi(y)^{4}+4 \beta \epsilon \phi(y)^{3}+A^{2}(y)-\phi_{1}(y)+\phi_{2}(y)+a \text {, } \\
& f_{5}(x)=6 \beta \phi(x)^{2}+2 \gamma \phi(x)+\delta \text {, } \\
& f_{6}(y)=6 \alpha \phi(y)^{2}+2 \epsilon \phi(y)+b \text {. }
\end{aligned}
$$

Here $a, b, c, d, \alpha, \beta, \gamma, \delta, \epsilon$ are arbitrary constants, $\phi, \phi_{1}, \phi_{2}$ are arbitrary additive maps, $\psi$ is an arbitrary nonzero exponential, $A^{2}$ is the diagonal of an arbitrary biadditive function and $\theta$ is any solution of (4.6).

Proof. From (FE), (FC) and (1.5) by Theorem 3.1, we get (3.2)-(3.6). Substitution of (3.2)-(3.6), respectively, into (FE) will yield the general solution of (FE) by Theorem 4.1. Figure 1 illustrates how the solutions $(5.1)-(5.5)$ are obtained from (3.2)-(3.6). The solution (3.2) when inserted into (FE) yields a special case of (1.4) after some manipulations and using solution (4.1) of Theorem 4.1, we get (5.1). In the diagram, this is indicated by a thick solid line joining from (3.2) to (5.1). The solution (3.3) yields, after some tedious manipulations, (5.2) and special cases of (5.1), (5.3), (5.4), and (5.5). These special cases are illustrated by thin solid lines joining one at a time from (3.3) to (5.1), (5.3), (5.4) and (5.5). The derivation of other solutions can be traced from the diagram in a similar manner. 
First, substitution of (1.5) and (3.2) with $\phi_{1}$ in place of $\phi$ into (FE) yields (after some rearrangement and using properties of $A^{2}(x)$ and $\phi_{1}$ )

$$
\begin{gathered}
{\left[f_{1}(x y)-\frac{1}{2} A^{2}(x y)\right]-\left[f_{1}\left(x y^{-1}\right)-\frac{1}{2} A^{2}\left(x y^{-1}\right)\right]} \\
=f_{4}(y)+c f_{6}(y)-A^{2}(y)+\phi_{1}(y)+b .
\end{gathered}
$$

By Theorem 4.1 with (1.5) and (3.2), we get (5.1). (This requires checking all solutions given in Theorem 4.1.)

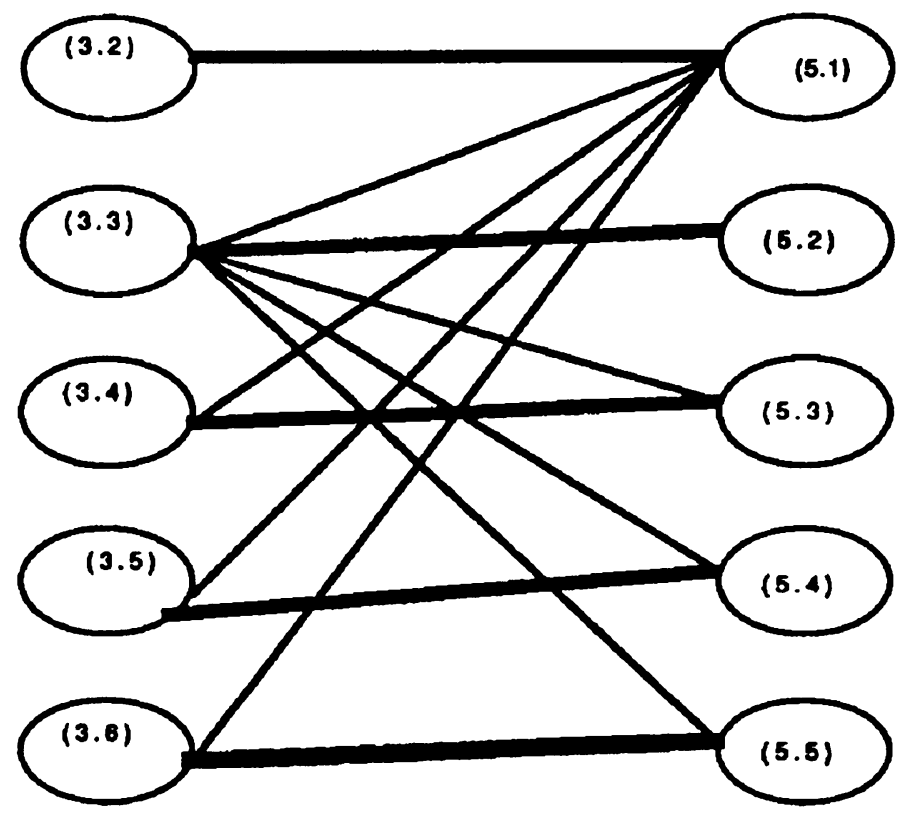

FIGURE 1

Substitution of (1.5) and (3.3) into (FE) yields

$$
\begin{aligned}
{\left[f_{1}(x y)\right.} & \left.-\frac{1}{2} A^{2}(x y)\right]-\left[f_{1}\left(x y^{-1}\right)-\frac{1}{2} A^{2}\left(x y^{-1}\right)\right] \\
& =\left[f_{4}(y)-A^{2}(y)+\phi(y)-a\right]+f_{5}(x)\left[f_{6}(y)-b\right] .
\end{aligned}
$$


By Theorem 4.1, (1.5) and (3.3), we get (5.2) and particular cases of (5.3)-(5.5) and (5.1).

Substitution of (1.5) and (3.4) into (FE) yields

$$
\begin{aligned}
f_{1}(x y)- & f_{1}\left(x y^{-1}\right)+\gamma\left[\alpha \psi\left(x y^{-1}\right)+\beta \psi\left(x y^{-1}\right)^{-1}\right]+A^{2}\left(x y^{-1}\right)+\phi\left(x y^{-1}\right)+d \\
= & (\gamma-b)\left[\alpha \psi(x)+\beta \psi(x)^{-1}\right]+A^{2}(x)+\phi(x)-a+b \delta+d+f_{4}(y) \\
& +\left[\alpha \psi(x)+\beta \psi(x)^{-1}-\delta\right] f_{6}(y)
\end{aligned}
$$

that is,

$$
\begin{aligned}
\left\{f_{1}(x y)\right. & \left.-\frac{1}{2} A^{2}(x y)-\frac{\gamma}{2}\left[\alpha \psi(x y)+\beta \psi(x y)^{-1}\right]\right\} \\
& -\left\{f_{1}\left(x y^{-1}\right)-\frac{1}{2} A^{2}\left(x y^{-1}\right)-\frac{\gamma}{2}\left[\alpha \psi\left(x y^{-1}\right)+\beta \psi\left(x y^{-1}\right)^{-1}\right]\right\} \\
= & f_{4}(y)-A^{2}(y)-\delta \frac{\gamma}{2}\left[\psi(y)+\psi(y)^{-1}\right]+\phi(y)-a+b \delta+\delta(\gamma-b) \\
+ & \left\{\alpha \psi(x)+\beta \psi(x)^{-1}-\delta\right\}\left\{f_{6}(y)-\frac{\gamma}{2}\left[\psi(y)+\psi(y)^{-1}\right]+(\gamma-b)\right\} .
\end{aligned}
$$

By Theorem 4.1, (1.5) and (3.4), we get (5.3) or a particular case of (5.1).

Substitution of (1.5) and (3.5) into (FE) and replacement of $\phi$ by $c \phi$ yield

$$
\begin{aligned}
f_{1}(x y)- & f_{1}\left(x y^{-1}\right)+\gamma \psi_{o}\left(x y^{-1}\right)\left[c \phi\left(x y^{-1}\right)+\alpha\right]+A^{2}\left(x y^{-1}\right)+\phi_{1}\left(x y^{-1}\right)+d \\
= & (\gamma-b) \psi_{o}(x)[c \phi(x)+\alpha]+A^{2}(x)+\phi_{1}(x)-a+b \delta+d \\
& +f_{4}(y)+\left\{\psi_{o}(x)[c \phi(x)+\alpha]-\delta\right\} f_{6}(y),
\end{aligned}
$$

that is,

$$
\begin{aligned}
\left\{f_{1}(x y)\right. & \left.-\frac{\gamma}{2} \psi_{o}(x y)[c \phi(x y)+\alpha]-\frac{1}{2} A^{2}(x y)\right\} \\
& -\left\{f_{1}\left(x y^{-1}\right)-\frac{\gamma}{2} \psi_{o}\left(x y^{-1}\right)\left[c \phi\left(x y^{-1}\right)+\alpha\right]-\frac{1}{2} A^{2}\left(x y^{-1}\right)\right\} \\
= & \left\{f_{4}(y)-\delta \gamma \psi_{o}(y)-A^{2}(y)+\phi_{1}(y)+\delta \gamma-a\right\} \\
& +\left\{\psi_{o}(x)[c \phi(x)+\alpha]-\delta\right\}\left\{f_{6}(y)-\gamma \psi_{o}(y)+\gamma-b\right\} .
\end{aligned}
$$

By Theorem 4.1, (1.5) and (3.5), we get (5.4) or a special case of (5.1).

Finally substitution of (1.5) and (3.6) into (FE) yields

$$
\begin{aligned}
f_{1}(x y) & -f_{1}\left(x y^{-1}\right)+\frac{3}{2} \alpha \beta \phi\left(x y^{-1}\right)^{4}+\alpha \gamma \phi\left(x y^{-1}\right)^{3}-3 \alpha \delta \phi\left(x y^{-1}\right)^{2} \\
& +A^{2}\left(x y^{-1}\right)+\phi_{1}\left(x y^{-1}\right)+d \\
= & \frac{3}{2} \alpha \beta \phi(x)^{4}+\alpha \gamma \phi(x)^{3}-3(\alpha \delta+\beta b) \phi(x)^{2}+A^{2}(x)-\gamma b \phi(x) \\
& +\phi_{1}(x)-a+\delta b+d+f_{4}(y)+\left\{3 \beta \phi(x)^{2}+\gamma \phi(x)-\delta\right\} f_{6}(y),
\end{aligned}
$$


that is,

$$
\begin{aligned}
& f_{1}(x y)- \frac{3}{4} \alpha \beta \phi(x y)^{4}-\frac{1}{2} \alpha \gamma \phi(x y)^{3}+ \\
&-\left\{f_{1}\left(x y^{-1}\right)-\frac{3}{2} \alpha \delta \phi(x y)^{2}-\frac{1}{2} A^{2}(x y)\right. \\
&\left.+\frac{3}{2} \alpha \delta \phi\left(x y^{-1}\right)^{4}-\frac{1}{2} A^{2}\left(x y^{-1}\right)\right\} \\
&=\left\{f_{4}(y)-\frac{3}{2} \alpha \beta \phi(y)^{4}-A^{2}(y)+\phi_{1}(y)-a\right\} \\
&+\left\{3 \beta \phi(x)^{2}+\gamma \phi(x)-\delta\right\}\left\{f_{6}(y)-3 \alpha \phi(y)^{2}-b\right\} .
\end{aligned}
$$

By Theorem 4.1, (1.5) and (3.6), we get either some particular cases of (5.1) or, more generally,

$$
\begin{aligned}
f_{1}(x)= & \frac{3}{4} \alpha \beta \phi(x)^{4}+\frac{1}{2}(\alpha \gamma+\beta \epsilon) \phi(x)^{3}-\left(\frac{3}{2} \alpha \delta-\frac{1}{4} \gamma \epsilon\right) \phi(x)^{2}+\frac{1}{2} A^{2}(x) \\
- & \frac{\delta}{2} \epsilon \phi(x)+\frac{1}{2} \phi_{2}(x)+\theta(x), \\
f_{2}(x)= & \frac{3}{4} \alpha \beta \phi(x)^{4}+\frac{1}{2}(\alpha \gamma-\beta \epsilon) \phi(x)^{3}-\left(\frac{3}{2} \alpha \delta+\frac{1}{4} \gamma \epsilon\right) \phi(x)^{2} \\
& +\frac{1}{2} A^{2}(x)+\frac{\delta \epsilon}{2} \phi(x)+\phi_{1}(x)-\frac{1}{2} \phi_{2}(x)-\theta(x)+d, \\
f_{3}(x)= & \frac{3}{2} \alpha \beta \phi(x)^{4}+\alpha \gamma \phi(x)^{3}-3(\alpha \delta+\beta b) \phi(x)^{2} \\
& +A^{2}(x)-b \gamma \phi(x)+\phi_{1}(x)-a+b \delta+d, \\
f_{4}(y)= & \frac{3}{2} \alpha \beta \phi(y)^{4}+\beta \epsilon \phi(y)^{3}+A^{2}(y)+\phi_{2}(y)-\phi_{1}(y)+a, \\
f_{5}(x)= & 3 \beta \phi(x)^{2}+\gamma \phi(x)-\delta, \\
f_{6}(y)= & 3 \alpha \phi(y)^{2}+\epsilon \phi(y)+b .
\end{aligned}
$$

This solution becomes (5.5), after replacing $\alpha$ by $2 \alpha, \beta$ by $2 \beta, \gamma$ by $2 \gamma, \epsilon$ by $2 \epsilon$, and $\delta$ by $-\delta$.

Since all the functions of $(5.1)-(5.5)$ satisfy (FE) and (FC) is satisfied by $f_{1}$ and $f_{2}$, the proof is complete.

Remark 5. In Theorems 3.1, 4.1 and 5.1 the complex field $\mathbf{C}$ may be replaced by any quadratically closed commutative field of characteristic different from 2 and 3.

\section{Solution of Swiatak's equation}

In this section, we present the general solution of Swiatak's functional equation

$$
f(x y)+f\left(x y^{-1}\right)=2 f(x)+2 f(y)+g(x) g(y),
$$

for $x, y \in \mathbf{G}$, where $f, g: \mathbf{G} \rightarrow \mathbf{K}$ are under (FC), $\mathbf{G}$ is an arbitrary group, and $\mathbf{K}$ is a quadratically closed (commutative) field of characteristic different from 2 and 3. 
Theorem 6.1. The general solution $f, g: \mathbf{G} \rightarrow \mathbf{K}$ of (6.1) where $f$ satisfies also (FC):

$$
f(t x y)=f(t y x)
$$

for all $t, x, y \in \mathbf{G}$ is given by the following list:

$$
\begin{gathered}
\left\{\begin{array}{l}
f(x)=A^{2}(x)-\frac{1}{2} c^{2}, \\
g(x)=c,
\end{array}\right. \\
\left\{\begin{array}{l}
f(x)=A^{2}(x)+c^{2}\left\{\psi(x)+\psi(x)^{-1}-2\right\}, \\
g(x)=c\left\{\psi(x)+\psi(x)^{-1}-2\right\}, \quad \psi \not \equiv 1,
\end{array}\right. \\
\left\{\begin{array}{l}
f(x)=A^{2}(x)+\frac{1}{12} c^{2} \phi(x)^{4}, \\
g(x)=c \phi(x)^{2},
\end{array}\right.
\end{gathered}
$$

where $A^{2}$ is the diagonal of an arbitrary biadditive function $A: \mathbf{G} \times \mathbf{G} \rightarrow \mathbf{K}$, $c$ is an arbitrary constant in $\mathbf{K}, \psi: \mathbf{G} \rightarrow \mathbf{K}$ is exponential, and $\phi: \mathbf{G} \rightarrow \mathbf{K}$ is additive.

Proof. It is easily verified that the sets of functions (6.3) - (6.5) satisfy (6.1) and (6.2). We prove the converse.

Equation (6.1) is a special case of equation (3.1) with

$$
p=q=2 f \quad \text { and } \quad h=g .
$$

Hence we can apply Theorem 3.1. We consider solutions (3.2) - (3.6) one by one.

First, solution (3.2) together with (6.6) yields immediately

$$
b=0, \quad h(y)=g(x)=c, \quad \text { and } \quad 2\{\phi(x)+d\}=-c^{2} .
$$

Thus $\phi=0, d=-\frac{1}{2} c^{2}$, and we have (6.3).

Similarly, (3.3) and (6.6) give

$$
g(x)=h(y)=2 b, \quad 2 b^{2}+a=0, \quad \text { and } \quad \phi(x)+d=a .
$$

Hence $\phi=0, d=-2 b^{2}$, and we again get (6.3) upon setting $c=2 b$.

Next, (3.4) together with (6.6) yields

$$
\begin{gathered}
b=0=a, \quad \gamma \delta=2 \gamma \alpha=2 \gamma \beta, \quad \phi(x)+d=-\gamma \delta, \\
\gamma=\alpha=\beta, \quad \text { and } \quad 2 \gamma=\delta .
\end{gathered}
$$

Therefore we have $\phi=0, d=-\gamma \delta$, and $\gamma=\alpha=\beta=\frac{1}{2} \delta$, whence $d=-2 \gamma^{2}$. Renaming $\gamma$ as $c$, we obtain the special case of (6.4) in which $\psi(x) \not \equiv \psi(x)^{-1}$ (cf. (3.4)).

Similarly, from (3.5) and (6.6) we get

$$
\begin{gathered}
b=0=a, \quad \phi=0, \quad \gamma \delta=\gamma \alpha, \quad \phi_{1}(x)+d=-\gamma \delta, \\
2 \gamma=\alpha, \quad \text { and } \quad 2 \gamma=\delta .
\end{gathered}
$$

Hence $\phi_{1}=0, \alpha=\delta=2 \gamma$, and $d=-2 \gamma^{2}$, so that we have

$$
\left\{\begin{array}{l}
f(x)=2 \gamma^{2} \psi_{o}(x)+A^{2}(x)-2 \gamma^{2} \\
g(x)=2 \gamma \psi_{o}(x)-2 \gamma
\end{array}\right.
$$


Defining $c:=\gamma$, and recalling that $\psi_{o}(x) \equiv \psi(x) \equiv \psi(x)^{-1} \not \equiv 1$ (cf. (3.5)), we again obtain a special case of (6.4). Combining the results of this paragraph and the preceding one, we now have the complete solution (6.4).

Finally, let us consider (3.6) with (6.6). We find that

$$
\begin{gathered}
\beta b=0, \quad-b \gamma \phi(x)-a+b \delta \equiv 0, \quad \alpha \gamma=\alpha \delta=0, \\
\phi_{1}(x)+d \equiv a, \quad \beta=2 \alpha, \quad \text { and } \quad \gamma \phi(x)-\delta \equiv 2 b .
\end{gathered}
$$

Thus $\phi_{1}=0, d=a=b \delta, \gamma \phi(x) \equiv 0$, and $\delta=-2 b$ follow. If $\phi=0$, then we have $f(x)=A^{2}(x)-2 b^{2}$ and $g(x)=2 b$, which is already included in (6.3). So we suppose now that $\phi \neq 0$, which means that $\gamma=0$. Also $\alpha \delta=0$, and if $\alpha=0$ we revert again to (6.3). Thus we suppose that $\alpha \neq 0$ and hence $\delta=0$. Therefore also $d=a=b=0$, and by virtue of $\beta=2 \alpha$ we have

$$
\left\{\begin{array}{l}
f(x)=3 \alpha^{2} \phi(x)^{4}+A^{2}(x) \\
g(x)=6 \alpha \phi(x)^{2}
\end{array}\right.
$$

which is $(6.5)$ with $c=6 \alpha$.

There are no more cases to consider, so the proof of the theorem is finished.

Corollary 6.2. The (Lebesgue) measurable solution $f, g: \Re \rightarrow \mathbf{C}$ of

$$
f(x+y)+f(x-y)=2 f(x)+2 f(y)+g(x) g(y),
$$

is given by

$$
\begin{gathered}
\left\{\begin{array}{l}
f(x)=a x^{2}-\frac{1}{2} c^{2}, \\
g(x)=c,
\end{array}\right. \\
\left\{\begin{array}{l}
f(x)=a x^{2}+c^{2}\left\{e^{\lambda x}+e^{-\lambda x}-2\right\}, \\
g(x)=c\left\{e^{\lambda x}+e^{-\lambda x}-2\right\},
\end{array}\right. \\
\left\{\begin{array}{l}
f(x)=a x^{2}+\frac{1}{12} c^{2} x^{4}, \\
g(x)=c x^{2},
\end{array}\right.
\end{gathered}
$$

for arbitrary complex constants $a, c, \lambda$.

Proof. Clearly, functions given by (6.7)-(6.9) satisfy (SE).

For the converse, we apply Theorem 6.1 with $(\mathbf{G}, \cdot)=(\Re,+)$, switching to additive notation, and consider the solutions (6.3) - (6.5) one at a time.

First, in solution (6.3) $f$ will be measurable if and only if the biadditive $A$, after symmetrization, is measurable. But then $A$ must be of the form $A(x, y)=a x y$ for some constant $a$. (Cf. [2], [13], for instance.) Thus $A^{2}(x)=A(x, x)=a x^{2}$ and we have (6.7).

If $g$ is measurable in (6.4), then $\psi$ must be measurable (and hence continuous; see [7], for example). The general nonzero measurable exponential $\psi: \Re \rightarrow \mathbf{C}$ is of the form $\psi(x)=e^{\lambda x}$ for arbitrary complex $\lambda$ (see [2], [13]). Now the measurability of $f$ and $\psi$ implies that of $A^{2}$, hence (6.4) becomes (6.8).

Finally, if $g: \Re \rightarrow \mathbf{C}$ is measurable in (6.5), then so is $\phi$. Since $\phi$ is additive, we have $\phi(x)=b x$ for some constant $b \in \mathbf{C}$. Again, the measurability 
of $f$ implies that of $A^{2}$. Renaming $c b^{2}$ as a new constant $c$, we have (6.9), and that completes the proof.

The following corollary is a straightforward consequence of Corollary 6.2.

Corollary 6.3. The general solution $f, g: \Re \rightarrow \Re$ of (6.1), among functions measurable on $\Re$ is given by

$$
\begin{gathered}
\left\{\begin{array}{l}
f(x)=a x^{2}-\frac{1}{2} c^{2}, \\
g(x)=c,
\end{array}\right. \\
\left\{\begin{array}{l}
f(x)=a x^{2}+2 c^{2}\{\cos \alpha x-1\}, \\
g(x)=2 c\{\cos \alpha x-1\},
\end{array}\right. \\
\left\{\begin{array}{l}
f(x)=a x^{2}+2 c^{2}\{\cosh \alpha x-1\}, \\
g(x)=2 c\{\cosh \alpha x-1\},
\end{array}\right. \\
\left\{\begin{array}{l}
f(x)=a x^{2}+\frac{1}{12} c^{2} x^{4}, \\
g(x)=c x^{2},
\end{array}\right.
\end{gathered}
$$

for arbitrary real constants $a, c, \alpha$.

Remark 8. Solutions (6.11) and (6.12) were omitted in Swiatak [12].

\section{Solution of a SPEcial case of Swiatak's equation on groups}

Next we shall consider the functional equation

$$
F(x y)+F\left(x y^{-1}\right)=2 F(x)+2 F(y)+\lambda F\left(x^{2}\right) F\left(y^{2}\right)
$$

for $F: \mathbf{G} \rightarrow \mathbf{K}$ (a commutative field), which is a special case of (FE). (Compare this with equation (1.1).)

In the following lemma, $\mathbf{K}^{*}:=\mathbf{K} \backslash\{0\}$ and $\operatorname{char} \mathbf{K} \neq 2$.

Lemma 7.1. Let $A^{2}$ be the diagonal of a biadditive function $A: \mathbf{G} \times \mathbf{G} \rightarrow \mathbf{K}$ and $\psi: \mathbf{G} \rightarrow \mathbf{K}^{*}$ be exponential. If

$$
A^{2}(x)=a\left\{\psi(x)+\psi(x)^{-1}-2\right\}+b\left\{\psi(x)^{2}+\psi(x)^{-2}-2\right\}
$$

for all $x \in \mathbf{G}$, then $A^{2}(x) \equiv 0$ and one of the following alternatives holds. Either

$$
a\left\{\psi(x)+\psi(x)^{-1}-2\right\}=b\left\{\psi(x)^{2}+\psi(x)^{-2}-2\right\}=0,
$$

or else $\mathbf{G}$ has a normal subgroup $\mathbf{S}$ of index 3 , the polynomial $u^{3}-1$ has three distinct roots $1, \omega, \omega^{2} \in \mathbf{K}^{*}, \psi$ is a morphism of $\mathbf{G}$ onto $\left\{1, \omega, \omega^{2}\right\}$ with kernel $\mathbf{S}$, and $a+b=0$. The converse also holds.

Proof. If $a=b=0$, then $A^{2}(x) \equiv 0$ and (7.2) is obvious. Henceforth we assume $(a, b) \neq(0,0)$. Since the diagonal of any biadditive map satisfies the parallelogram law, we deduce from (7.1) that

$$
\begin{aligned}
a\{\psi(x y) & \left.+\psi(x y)^{-1}-2\right\}+b\left\{\psi(x y)^{2}+\psi(x y)^{-2}-2\right\} \\
& +a\left\{\psi\left(x y^{-1}\right)+\psi\left(x y^{-1}\right)^{-1}-2\right\}+b\left\{\psi\left(x y^{-1}\right)^{2}+\psi\left(x y^{-1}\right)^{-2}-2\right\} \\
= & 2 a\left\{\psi(x)+\psi(x)^{-1}-2\right\}+2 b\left\{\psi(x)^{2}+\psi(x)^{-2}-2\right\} \\
& +2 a\left\{\psi(y)+\psi(y)^{-1}-2\right\}+2 b\left\{\psi(y)^{2}+\psi(y)^{-2}-2\right\}
\end{aligned}
$$


holds for all $x, y \in \mathbf{G}$. Simplifying, using the fact that $\psi$ is exponential, and multiplying through by $\psi(x)^{2} \psi(y)^{2}$ to clear inverses, we get

$$
\begin{aligned}
& a\left\{\psi(x)^{3} \psi(y)^{3}+\psi(x) \psi(y)\right\}+b\left\{\psi(x)^{4} \psi(y)^{4}\right\} \\
&+a\left\{\psi(x)^{3} \psi(y)+\psi(x) \psi(y)^{3}\right\}+b\left\{\psi(x)^{4}+\psi(y)^{4}\right\} \\
&= 2 a\left\{\psi(x)^{3} \psi(y)^{2}+\psi(x) \psi(y)^{2}\right\}+2 b\left\{\psi(x)^{4} \psi(y)^{2}+\psi(y)^{2}\right\} \\
&+2 a\left\{\psi(x)^{2} \psi(y)^{3}+\psi(x)^{2} \psi(y)-2 \psi(x)^{2} \psi(y)^{2}\right\} \\
&+2 b\left\{\psi(x)^{2} \psi(y)^{4}+\psi(x)^{2}-2 \psi(x)^{2} \psi(y)^{2}\right\} .
\end{aligned}
$$

Consider (7.3) at $y=x$. If $b \neq 0$, then we have a nontrivial polynomial relation (of degree 8) in $\psi(x)$, hence $|\psi(\mathbf{G})| \leq 8$. If $b=0$, then $a \neq 0$ by hypothesis and we have a nontrivial polynomial relation (of degree 6) in $\psi(x)$, and so $|\psi(\mathbf{G})| \leq 6$. In either case, we have

$$
|\psi(\mathbf{G})|=m \quad \text { (finite) }
$$

Thus $\psi(\mathbf{G})$ is a multiplicative subgroup in $\mathbf{K}^{*}$ of order $m$, so

$$
\psi(x)^{m}=1 \quad \text { for all } x \in \mathbf{G} .
$$

It is easy to show that $p=$ char $\mathbf{K}$ does not divide $m$. For this purpose, let $p \neq 0$. Then the mapping $u \mapsto u^{p}$ is an injective field morphism. Hence $u^{p}=1$ has $u=1$ as the unique solution. If we had $m=p q$, then it would follow from $u^{m}=u^{p q}=\left(u^{q}\right)^{p}$ that $u^{m}=1$ if and only if $u^{q}=1$. But the latter cannot carry $m(>q)$ distinct roots, contrary to (7.4) (see Jacobson [6], p. 95). Therefore $p$ does not divide $m$.

Moreover, from (7.1) and (7.5), it follows that

$$
\begin{aligned}
m^{2} A(x, x) & =A^{2}\left(x^{m}\right) \\
& =a\left\{\psi(x)^{m}+\psi(x)^{-m}-2\right\}+b\left\{\psi(x)^{2 m}+\psi(x)^{-2 m}-2\right\} \\
& =0 .
\end{aligned}
$$

This proves that $A^{2}(x) \equiv 0$, since char $\mathbf{K}$ does not divide $m$.

Now (7.1) reduces to

$$
a\left\{\psi(x)+\psi(x)^{-1}-2\right\}+b\left\{\psi(x)^{2}+\psi(x)^{-2}-2\right\}=0 .
$$

If $a b=0$, then (7.6) implies (7.2). So from this point, we assume that $a \neq 0$ and $b \neq 0$. Observe that

$$
\psi(x)+\psi(x)^{-1}-2=\{\psi(x)-1\}^{2} \psi(x)^{-1} \quad \text { for all } x \in \mathbf{G},
$$

so we have

$$
\psi(x)+\psi(x)^{-1}-2=0 \text { if and only if } x \in \operatorname{Ker} \psi .
$$

From (7.6) we deduce that (since $a \neq 0 \neq b$ )

$$
x \in \operatorname{Ker} \psi \quad \text { if and only if } \quad x^{2} \in \operatorname{Ker} \psi .
$$

Observe that

$$
\left\{\psi(x)+\psi(x)^{-1}-2\right\}^{2}=\left\{\psi\left(x^{2}\right)+\psi\left(x^{2}\right)^{-1}-2\right\}-4\left\{\psi(x)+\psi(x)^{-1}-2\right\},
$$

so (7.6) can be put in the form

$$
\left\{\psi(x)+\psi(x)^{-1}-2\right\}\left\{b\left(\psi(x)+\psi(x)^{-1}-2\right)+(4 b+a)\right\}=0 .
$$


Hence $\psi(x)+\psi(x)^{-1}-2$ has at most two distinct values, one of which is 0 . By (7.7) then, $\psi$ has at most three distinct values, one of which is 1 . That is, $m=|\psi(\mathbf{G})| \leq 3$.

If $m=1$, then $\psi=1$ and (7.2) follows. If $m=2$, then (by (7.5)) $\psi(x)^{2}=1$ for all $x \in \mathbf{G}$. That is, $x^{2} \in \operatorname{Ker} \psi$ and, by (7.8), $x \in \operatorname{Ker} \psi$. But then $\psi=1$, contradicting $|\psi(\mathbf{G})|=2$.

Finally, we consider $m=3$. Let $\psi(\mathbf{G})=\left\{1, \omega, \omega^{2}\right\}$, where these are the three distinct cube roots of 1 . Also, $\mathbf{S}=\operatorname{Ker} \psi$ is a normal subgroup of G with index 3. Pick $x_{o}$ with $\psi\left(x_{o}\right)=\omega$ and put $x=x_{o}$ in (7.6). Then $a\left(\omega+\omega^{2}-2\right)+b\left(\omega^{2}+\omega-2\right)=0$, which simplifies to $(a+b)(-3)=0$. Thus, since char $\mathbf{K}$ does not divide 3 , we have $a+b=0$.

Conversely, (7.2) together with $A=0$ obviously implies (7.1). For the other part, suppose $\mathbf{G}$ has a normal subgroup $\mathbf{S}$ of index 3 , and that $u^{3}-1$ has distinct roots $1, \omega, \omega^{2}$ in $\mathbf{K}^{*}$. Then there exists a morphism $\psi: \mathbf{G} \rightarrow$ $\left\{1, \omega, \omega^{2}\right\}$ with $\operatorname{Ker} \psi=\mathbf{S}$. If $a+b=0$ and $A=0$, then it is easy to verify that (7.1) holds. This completes the proof of the lemma.

Now we determine the general solution $F: \mathbf{G} \rightarrow \mathbf{K}$ of the equation (SEs).

Theorem 7.2. Let $\mathbf{G}$ be a group, $\mathbf{K}$ a commutative quadratically closed field of characteristic different from 2 and 3 . Then the general solution $F: \mathbf{G} \rightarrow \mathbf{K}$ of (SEs) satisfying

$$
F(t x y)=F(t y x) \quad(t, x, y \in \mathbf{G})
$$

is given by

$$
F(x)=A^{2}(x) \quad(x \in \mathbf{G}),
$$

if $\lambda=0$; and by one of the three forms

$$
\begin{gathered}
F(x)=0 \quad(x \in \mathbf{G}), \\
F(x)=-2 \lambda^{-1} \quad(x \in \mathbf{G}),
\end{gathered}
$$

or

$$
F(x)=-3 \lambda^{-1} \chi_{\mathbf{G} \backslash \mathbf{S}}(x) \quad(x \in \mathbf{G}),
$$

if $\lambda \neq 0$. Here $A^{2}$ is the diagonal of an arbitrary biadditive map, $\chi_{D}: \mathbf{G} \rightarrow \mathbf{K}$ denotes the characteristic function of the set $D \subset \mathbf{G}$, and solution (7.12) arises only if $\mathbf{G}$ has a normal subgroup $\mathbf{S}$ of index 3 and the polynomial $u^{3}-1$ has three distinct roots in $\mathbf{K}$.

Proof. If $\lambda=0$, then (SEs) reduces to the well known quadratic functional equation

$$
F(x y)+F\left(x y^{-1}\right)=2 F(x)+2 F(y) \quad(x, y \in \mathbf{G}) .
$$

Its general solution (see [1], Lemma 2) under the factorization condition (FC) is given by (7.9) for arbitrary biadditive $A: \mathbf{G}^{2} \rightarrow \mathbf{K}$.

Henceforth, we assume that $\lambda \neq 0$. Now we consider (SEs) as a special case of (3.1) and apply Theorem 6.1, making special use of the connections

$$
f=F, \quad g(x)=\sqrt{\lambda} F\left(x^{2}\right) .
$$

We treat solutions $(6.3)-(6.5)$ one by one. 
First, we consider solution (6.3). We have

$$
F(x)=f(x)=A^{2}(x) \frac{1}{2} c^{2}, \quad \text { and } \sqrt{\lambda} F\left(x^{2}\right)=g(x)=c .
$$

So comparison of these two yields $A^{2}\left(x^{2}\right)-\frac{1}{2} c^{2}=c \lambda^{-\frac{1}{2}}$, or, by the morphism property of $A^{2}$,

But this implies that

$$
4 A^{2}(x) c \lambda^{-\frac{1}{2}}+\frac{1}{2} c^{2}=c\left\{\lambda^{-\frac{1}{2}}+\frac{c}{2}\right\} .
$$

$$
A^{2}(x) \equiv 0=c\left\{\lambda^{-\frac{1}{2}}+\frac{c}{2}\right\},
$$

from which we deduce (cf. (7.15)) that $F$ is constant. But it is easy to see that the only constant solutions of (SEs) are given by (7.10) and (7.11).

Second, consider (6.4). Connection (7.14) yields in particular

$$
\left\{\begin{array}{c}
2 F(x)=f(x)=A^{2}(x)+c^{2}\left\{\psi(x)+\psi(x)^{-1}-2\right\}, \\
F\left(x^{2}\right)=\lambda^{-\frac{1}{2}} g(x)=c \lambda^{-\frac{1}{2}}\left\{\psi(x)+\psi(x)^{-1}-2\right\} .
\end{array}\right.
$$

This implies

$$
c^{2}\left\{\psi(y)^{2}+\psi(y)^{-2}-2\right\}+4 A^{2}(y)=c \lambda^{-\frac{1}{2}}\left\{\psi(y)+\psi(y)^{-1}-2\right\},
$$

for all $y \in \mathbf{G}$. Here we have used the facts that $\psi\left(y^{2}\right)=\psi(y)^{2}$ and $A^{2}\left(y^{2}\right)=$ $A\left(y^{2}, y^{2}\right)=4 A(y, y)=4 A^{2}(y)$. Now we apply Lemma 7.1 to (7.17). Thus we have

$$
A^{2}(x) \equiv 0
$$

and one of two alternatives. One possibility is

$$
c^{2}\left\{\psi(y)^{2}+\psi(y)^{-2}-2\right\}=c \lambda^{-\frac{1}{2}}\left\{\psi(y)+\psi(y)^{-1}-2\right\}=0,
$$

in which case (7.16) and (7.18) again lead to constant $F$.

On the other hand, the alternative is that $\mathbf{G}$ has a normal subgroup $\mathbf{S}$ of index 3 , the polynomial $u^{3}-1$ has three distinct roots $1, \omega, \omega^{2}$ in $\mathbf{K}, \psi$ is a morphism of $\mathbf{G}$ onto $\left\{1, \omega, \omega^{2}\right\}$ with kernel $\mathbf{S}$, and $c \lambda^{-\frac{1}{2}}-c^{2}=0$. This last equation means that either $c=0$ or $c=\lambda^{-\frac{1}{2}}$. If $c=0$, then (7.16) and (7.18) again yield constancy of $F$. So let us explore the option

$$
c=\lambda^{-\frac{1}{2}} \text {. }
$$

Since $\psi$ has kernel $\mathbf{S}$, we have $\psi(y)+\psi(y)^{-1}-2=0$ if $y \in \mathbf{S}$, and $\psi(y)+$ $\psi(y)^{-1}-2=\omega+\omega^{2}-2=-3$ if $y \in \mathbf{G} \backslash \mathbf{S}$. Hence

$$
\psi(y)+\psi(y)^{-1}-2=-3 \chi_{\mathbf{G} \backslash \mathbf{S}}(y) .
$$

Using this with (7.19) and (7.18) in (7.16), we have

$$
F(y)=-3 \lambda^{-1} \chi_{\mathbf{G} \backslash \mathbf{S}}(y), \quad y \in \mathbf{G},
$$

which is (7.12). It is easily checked that (7.12) satisfies (SEs) by doing a caseby-case analysis according to the possible locations of $x$ and $y$ with respect to S.

Finally, consider (6.5). Here (7.14) gives

$$
\left\{\begin{array}{l}
2 F(x)=f(x)=A^{2}(x)+\frac{1}{12} c^{2} \phi(x)^{4}, \\
F\left(x^{2}\right)=\lambda^{-\frac{1}{2}} g(x)=c \lambda^{-\frac{1}{2}} \phi(x)^{2} .
\end{array}\right.
$$


As before, we have (since $\phi\left(x^{2}\right)=2 \phi(x)$ by $(3.8)$ )

$$
4 A^{2}(x)+\frac{4}{3} c^{2} \phi(x)^{4}=c \lambda^{-\frac{1}{2}} \phi(x)^{2} .
$$

The second term of (7.21) is of degree 4 in $x$, while the other two terms are of degree 2. Hence (7.21) implies that

$$
c^{2}=0 \quad \text { and } \quad A^{2}(x)=\frac{1}{4} c \lambda^{-\frac{1}{2}} \phi(x)^{2} .
$$

Thus, we have $c=0=A^{2}$, and from (7.20) it follows that $F=0$ again. This exhausts all cases and concludes the proof of Theorem 7.2.

We end this section with the following remarks.

Remark 6. If $\mathbf{G}$ has no normal subgroup of index 3 , or if $\mathbf{K}$ does not have three distinct cube roots of unity, then Theorem 7.2 shows that the only solutions of (SEs) are the (7.9) quadratic ones, if $\lambda=0$, and the two constant solutions (7.10) and (7.11), if $\lambda \neq 0$. This will be the case, in particular, if $\mathbf{G}=\left(\Re^{n},+\right)$ or if $\mathbf{K}=\Re$.

Remark 7. For the general solution of (SEs) on a restricted domain, as in the original application of Lau [9], see [3].

\section{ACKNOWLEDGMENT}

This research was supported by grants from the College of Arts and Sciences of the University of Louisville and from NSERC of Canada.

\section{REFERENCES}

1. J. Aczél, J. K. Chung and C. T. Ng, Symmetric second differences in product form on groups, Topics in Mathematical Analysis (Th. M. Rassias, ed.), World Scientific Press, Singapore, 1986, pp. 1-22.

2. J. Aczél and J. Dhombres, Functional equations in several variables, Cambridge University Press, Cambridge, 1988.

3. J. K. Chung, B. R. Ebanks, C. T. Ng and P. K. Sahoo, On a functional equation connected with Rao's quadratic entropy, Proc. Amer. Math. Soc. 120 (1994), 843-848.

4. J. K. Chung, P. L. Kannappan and C. T. Ng, A generalization of the cosine-sine functional equation on groups, Linear Algebra Appl. 66 (1985), 259-277.

5. B. R. Ebanks, P. L. Kannappan and P. K. Sahoo, A common generalization of functional equations characterizing normed and quasi-inner-product spaces, Canad. Math. Bull. 35 (1992), 321-327.

6. N. Jacobson, Lectures in abstract algebra, Vol. III-Theory of fields and Galois theory, Van Nostrand, Princeton, NJ, 1964.

7. P. L. Kannappan, The functional equation $f(x y)+f\left(x y^{-1}\right)=2 f(x) f(y)$ for groups, Proc. Amer. Math. Soc. 29 (1968), 69-74.

8. E. L. Koh, The Cauchy functional equations in distributions, Proc. Amer. Math. Soc. 106 (1989), 641-646.

9. K. S. Lau, Characterization of Rao's quadratic entropies, Sankhya A 47 (1985), 295-309.

10. R. C. Penney and A. L. Rukhin, D'Alembert functional equation on groups, Proc. Amer. Math. Soc. 77 (1979), 73-80.

11. A. L. Rukhin, The solution of the functional equation of D'Alembert's type for commutative groups, Internat. J. Math. Sci. 5 (1982), 315-355. 
12. H. Swiatak, On two functional equations connected with the equation $\phi(x+y)+\phi(x-y)=$ $2 \phi(x)+2 \phi(y)$, Aequationes Math. 5 (1970), 3-9.

13. L. Székelyhidi, Convolution type functional equations on topological abelian groups, World Scientific, Singapore, 1991.

14. W. H. Wilson, On certain related functional equations, Bull. Amer. Math. Soc. 26 (1920), 300-312.

(J. K. Chung) Department of Applied Mathematics, South China University of Technology, Guanzhou, People's Republic of China

(B. R. Ebanks and P. K. Sahoo) Department of Mathematics, University of Louisville, LOUISVILLE, KENTUCKY 40292

E-mail address, B. R. Ebanks: breban01@ulkyvx.louisville.edu

E-mail address, P. K. Sahoo: pksaho01@homer.louisville.edu

(C. T. Ng) Department of Pure Mathematics, University of Waterloo, Waterloo, ONTARIO, N2L 3G1, CANADA

E-mail address: ctng@watdragon. uwaterloo.ca 